\title{
An Iterative Method for a Common Solution of a Combination of the Split Equilibrium Problem, a Finite Family of Nonexpansive Mapping and a Combination of Variational Inequality Problem
}

\author{
Ihssane Hay, Abdellah Bnouhachem and Themistocles M.Rassias
}

\begin{abstract}
The present paper aims to deal with an iterative algorithm for finding common solution of the combination of the split equilibrium problem and a finite family of nonexpansive mappings and the combination of variational inequality problem in the setting of real Hilbert spaces. Further, we prove that the sequences generated by the proposed iterative method converge strongly to a common solution to these problems. A numerical example is presented to illustrate the proposed method and convergence result. The results and method presented in this paper generalize, extend and unify some known results in the literatures.
\end{abstract}

\section{Introduction.}

The theory of equilibrium problems has grown enormously in various branches of the pure and applied sciences, it has been widely studied in the literature. It provides a framework for many problems in finance, economics, networks analysis, optimization and others; see for example [2, $4,5,6,7,8,10,11,12,18,19,20,21,22,26]$.

Let $\mathcal{H}$ be a real Hilbert space with the inner product $<.>$ and the norm $\|$. $\|$. Let $\mathcal{C}$ be nonempty closed convex subset of Hilbert space $\mathcal{H}$. Given a bifunction $F: \mathcal{C} \times \mathcal{C} \longrightarrow \mathcal{H}$, the standard equilibrium problem is formulated as follows:

$$
\left\{\begin{array}{l}
\text { Find } \quad x^{*} \in \mathcal{C} \\
F\left(x^{*}, x\right) \geq 0, \quad \forall x \in \mathcal{C},
\end{array}\right.
$$

which was first considered and investigated by Blum and Oettli [1]. The solution set of the equilibrium problem is denoted by $E P(F)$.

Inspired by a wide variety of works in this direction, Kazmi and Rivzi [17] have recently investigated and studied a new form of the equilibrium problem called the split equilibrium problem:

Key words and phrases. Split equilibrium problem; variational inequality problem; fixed point problem; projection method; nonexpansive mapping. 2010 Mathematics Subject Classification. 49J30, 47H09, 47J20.

Corresponding author: Abdellah Bnouhachem. 
let $\mathcal{C}$ and $\mathcal{Q}$ be nonempty closed convex subsets of Hilbert spaces $\mathcal{H}_{1}$ and $\mathcal{H}_{2}$, respectively. Given two bifunctions $F: \mathcal{C} \times \mathcal{C} \longrightarrow \mathcal{H}_{1}$, and $G: \mathcal{Q} \times \mathcal{Q} \longrightarrow \mathcal{H}_{2}$ and a bounded linear operator $A: \mathcal{H}_{1} \longrightarrow \mathcal{H}_{2}$, the split equilibrium problem is defined by:

$$
\left\{\begin{array}{l}
\text { Find } \quad x^{*} \in \mathcal{C} \\
F\left(x^{*}, x\right) \geq 0, \quad \forall x \in \mathcal{C},
\end{array}\right.
$$

and

$$
\left\{\begin{array}{l}
\text { Find } \quad y^{*}=A x^{*} \in \mathcal{Q} \\
G\left(y^{*}, y\right) \geq 0, \quad \forall y \in \mathcal{Q} .
\end{array}\right.
$$

If $G \equiv 0$ and $F\left(x^{*}, x\right)=\left\langle B x^{*}, x-x^{*}\right\rangle$, where $B: \mathcal{C} \longrightarrow \mathcal{H}$, is a nonlinear operator, then the split equilibrium problem collapses to the classical variational inequality problem:

$$
\left\{\begin{array}{l}
\text { Find } \quad x^{*} \in \mathcal{C} \\
\left\langle B x^{*}, x-x^{*}\right\rangle \geq 0, \quad \forall x \in \mathcal{C} .
\end{array}\right.
$$

The set of solutions of $(1.4)$ is denoted $(\mathcal{V I P})_{B, \mathcal{C}}$. It is easy to observe that

$$
x^{*} \in(\mathcal{V I P})_{B, \mathcal{C}} \Longleftrightarrow x^{*}=P_{C}\left[x^{*}-\rho B x^{*}\right], \text { where } \rho>0 \text {. }
$$

Variational inequalities are being used as a mathematical programming tool in modeling a large class of problems arising in various branches of pure and applied sciences. In recent years, variational inequalities have been generalized and extended novel and new techniques in several directions. We now have a variety of techniques to suggest and analyze various iterative algorithms for solving variational inequalities and related optimization problems; see [1-28].

Both classes of problems; variational inequality and split equilibrium problem, have been studied and treated in details in several research works due to its important role on the development of many problems; see for example $[3,9,13,14,15,16,17,28]$.

In the present paper, motivated by the above works and related literature, we present a new iterative algorithm for finding a common element of the solution set of common fixed points of a finite family of nonexpansive mappings and the solution set of a combination of the split equilibrium problem and the solution set of a combination of variational inequality problem. More precisely, we use the idea of combination of the split equilibrium problem. According to numerical results, the iteration algorithm for the combination of the split equilibrium problem converges faster than the iterative algorithm of the split equilibrium problem. Under appropriate conditions we derive the strong convergence results for this method. Preliminary numerical experiments are included to verify the theoretical assertions of the proposed method. Since the combination of the split equilibrium problem includes the split equilibrium problem and the equilibrium problem as special cases, results presented in this paper continue to hold for these problems. 


\section{Preliminaries.}

Let $\mathcal{H}_{1}$ and $\mathcal{H}_{2}$ be two real Hilbert spaces with inner product $<$. $>$, and norm $\|$. $\|$. Let $\mathcal{C}$ and $Q$ be nonempty closed convex subsets of Hilbert spaces $\mathcal{H}_{1}$ and $\mathcal{H}_{2}$, respectively. For $i \in\{1, \ldots, N\}$, let $\sum_{i=1}^{N} a_{i} F_{i}: \mathcal{C} \times \mathcal{C} \longrightarrow \mathcal{H}_{1}$, and $\sum_{i=1}^{N} b_{i} G_{i}: \mathcal{Q} \times \mathcal{Q} \longrightarrow \mathcal{H}_{2}$, be two bifunctions, and let $\sum_{i=1}^{N} c_{i} B_{i}: \mathcal{H}_{1} \longrightarrow \mathcal{H}_{1}$, with $\left.a_{i} \in\right] 0,1\left[, b_{i} \in\right] 0,1\left[, c_{i} \in\right] 0,1\left[, \sum_{i=1}^{N} a_{i}=\sum_{i=1}^{N} b_{i}=\right.$ $\sum_{i=1}^{N} c_{i}=1$ and $A: \mathcal{H}_{1} \longrightarrow \mathcal{H}_{2}$, be a bounded linear operator. The combination of split equilibrium problem (CSEP) is defined as:

$$
\left\{\begin{array}{l}
\text { Find } \quad x^{*} \in \mathcal{C} \\
\sum_{i=1}^{N} a_{i} F_{i}\left(x^{*}, x\right) \geq 0, \quad \forall x \in \mathcal{C}
\end{array}\right.
$$

and

$$
\left\{\begin{array}{l}
\text { Find } \quad y^{*}=A x^{*} \in \mathcal{Q} \\
\sum_{i=1}^{N} b_{i} G_{i}\left(y^{*}, y\right) \geq 0, \quad \forall y \in Q .
\end{array}\right.
$$

The solution set of the (CSEP) is denoted by $\Omega=\left\{p \in \mathcal{C} ; z \in \cap_{i=1}^{N} \operatorname{EP}\left(F_{i}\right)\right.$ such that $A p \in$ $\left.\cap_{i=1}^{N} E P\left(G_{i}\right)\right\}$.

If $F_{i}=F$ and $G_{i}=G, \forall i=1,2, \ldots, N$, then the combination of split equilibrium problem (2.1)-(2.2) reduces to the split equilibrium problem (1.2)-(1.3). The fixed point problem for a sequence of nonexpansive mappings $S_{n}: \mathcal{C} \longrightarrow \mathcal{C}$ is to find $x \in C$ such that

$$
S_{n} x=x
$$

The set of all fixed points of $S_{n}$ is denoted by $F\left(S_{n}\right)$. The combination of variational inequality problem (CVIP) is defined as:

$$
\left\{\begin{array}{l}
\text { Find } \quad x^{*} \in \mathcal{C} \\
\left\langle\sum_{i=1}^{N} c_{i} B_{i}\left(x^{*}\right), x-x^{*}\right\rangle \geq 0, \quad \forall x \in \mathcal{C} .
\end{array}\right.
$$

The solution set of the (CVIP) is denoted by $(\mathcal{V I P})_{\sum_{i=1}^{N} c_{i} B_{i}, \mathcal{C}}$

We introduce the following definitions, which are useful in the following analysis.

Definition 1. Let $\mathcal{C}$ be a nonempty closed convex subset of $\mathbb{R}^{n}$, and $v \in \mathbb{R}^{n}$, then the projection of $v$ onto $\mathcal{C}$ is denoted by $P_{\mathcal{C}}(v)$, that is,

$$
P_{\mathcal{C}}(v):=\arg \min \{\|v-u\| / u \in \mathcal{C}\} .
$$

Since $\mathcal{C}$ is convex and closed, the projection onto $\mathcal{C}$ is unique.

Definition 2. The mapping $T: \mathcal{C} \longrightarrow \mathcal{C}$ is said to be : 
1) strongly positive if $\langle T x, x\rangle \geq \rho\|x\|^{2}$, for all $x \in \mathcal{H}$, where $\rho$ is a positive constant.

2) $\beta$-inverse strongly monotone over $\mathcal{C}$, if there exists a positive real number $\beta>0$ such that

$$
\langle T(u)-T(v), u-v\rangle \geq \beta\|T(u)-T(v)\|^{2} ; \quad \forall u, v \in \mathcal{C},
$$

3) nonexpansive if

$$
\|T x-T y\| \leq\|x-y\| ; \quad \forall x, y \in \mathcal{C} .
$$

4) lower semicontinuous at $x_{0} \in \mathcal{C}$ if, for any sequence $\left\{x_{n}\right\}$ in $\mathcal{C}$ with $x_{n} \longrightarrow x_{0}$,

$$
T\left(x_{0}\right) \leq \lim _{n \rightarrow \infty} T\left(x_{n}\right) .
$$

We list some fundamental lemmas that are useful in the consequent analysis. The first lemma provides some basic properties of projection onto $\mathcal{C}$.

Lemma 2.1. (i). If $T$ is nonexpansive, then $I-T$ is 1 - inverse strongly monotone;

(ii). If $T: \mathcal{C} \longrightarrow \mathcal{C}$, is $\beta$-inverse strongly monotone, then for all $\lambda \in] 0,2 \beta[, I-\lambda T$ is nonexpansive.

Lemma 2.2. Let $\mathcal{C} \subset \mathbb{R}^{n}$, be a closed convex set, then we have

1) $\forall v \in \mathbb{R}^{n}, \forall w \in \mathcal{C}$

$$
\left\langle v-P_{\mathcal{C}}(v), w-P_{\mathcal{C}}(v)\right\rangle \leq 0
$$

2) $\forall v \in \mathbb{R}^{n}, \forall w \in \mathbb{R}^{n}$

$$
\left\|P_{\mathcal{C}}(v)-P_{\mathcal{C}}(w)\right\| \leq\|v-w\|
$$

3) $\forall v \in \mathbb{R}^{n}, \forall u \in \mathcal{C}$

$$
\left\|P_{\mathcal{C}}(v)-u\right\| \leq\|v-u\|
$$

Lemma 2.3. It is well known that in case of a real Hilbert space $\mathcal{H}$, the following assertions:
(i) $\langle x, y\rangle=\frac{1}{2}\left(\|x\|^{2}+\|y\|^{2}-\|x-y\|^{2}\right)$,
(ii) $\|\alpha x+(1-\alpha) y\|^{2}=\alpha\|x\|^{2}+(1-\alpha)\|y\|^{2}-\alpha(1-\alpha)\|x-y\|^{2}$,

hold for all $\alpha \in[0,1]$ and $x, y \in \mathcal{H}$, such that $x \neq y$. 
It is well known that every nonexpansive operator $T: H \rightarrow H$ satisfies, for all $(x, y) \in$ $H \times H$, the inequality

$$
\langle(x-T x)-(y-T y), T y-T x\rangle \leq \frac{1}{2}\|(T x-x)-(T y-y)\|^{2}
$$

and therefore, we get, for all $(x, y) \in H \times F(T)$,

$$
\langle x-T x, y-T x\rangle \leq \frac{1}{2}\|T x-x\|^{2} .
$$

We assume that both $F: \mathcal{C} \times \mathcal{C} \longrightarrow \mathcal{H}_{1}$ and $G: \mathcal{Q} \times \mathcal{Q} \longrightarrow \mathcal{H}_{2}$ are bifunctions satisfying the following assumptions:

\section{Assumption 2.1}

1) $F(x, x)=0 \quad \forall x \in \mathcal{C}$;

2) $F$ is monotone i.e., $F(x, y)+F(y, x) \leq 0 \quad \forall x, y \in \mathcal{C}$;

3) $F$ is hemicontinuous i.e.

$$
\forall x, y, z \in \mathcal{C} \quad \lim _{t \rightarrow 0} F(t z+(1-t) x, y) \leq F(x, y)
$$

4) for each $x \in \mathcal{C}, y \longrightarrow F(x, y)$ is convex and lower semicontinuous mapping.

Lemma 2.4. [1] Let $\mathcal{C}$ be a nonempty closed convex subset of $\mathcal{H}$ and let $F$ be a function which satisfies above assumptions, then for each $x \in \mathcal{H}$, for $r>0$, there exists $z \in \mathcal{C}$ such that

$$
F(z, y)+\frac{1}{r}\langle y-z, z-x\rangle \geq 0 \quad \forall y \in \mathcal{C}
$$

Moreover, define a mapping $T_{r}^{F}: \mathcal{C} \longrightarrow \mathcal{H}$ as follows:

$$
T_{r}^{F}(x)=\left\{z \in \mathcal{C}: F(z, y)+\frac{1}{r}\langle y-z, z-x\rangle \geq 0 \quad \forall y \in \mathcal{C}\right\} .
$$

Then for all $x \in \mathcal{H}$, we have the following :

(i) $T_{r}^{F}$ is single-valued;

(ii) $T_{r}^{F}$ is firmly nonexpansive, i.e., for all $x, y \in \mathcal{H}$

$$
\left\|T_{r}^{F}(x)-T_{r}^{F}(y)\right\|^{2} \leq\left\langle T_{r}^{F}(x)-T_{r}^{F}(y), x-y\right\rangle ;
$$

(iii) $F\left(T_{r}^{F}\right)=E P(F)$;

(iv) $E P(F)$ is closed and convex. 
Lemma 2.5. [25] Let $\mathcal{C}$ be a nonempty closed convex subset of a real Hilbert space $\mathcal{H}$. For every $i \in$ $\{1, \ldots, N\}$, let $F_{i}: \mathcal{C} \times \mathcal{C} \longrightarrow \mathcal{H}$ be a bifunction satisfying assumptions 2.1 , with $\cap_{i=1}^{N} E P\left(F_{i}\right) \neq \emptyset$. Then for $a_{i} \in(0,1)$ and $\sum_{i=1}^{N} a_{i}=1$, we conclude that $\sum_{i=1}^{N} a_{i} F_{i}$, also satisfies the same above assumptions, and

$$
F\left(T_{r}\right)=E P\left(\sum_{i=1}^{N} a_{i} F_{i}\right)=\cap_{i=1}^{N} E P\left(F_{i}\right) .
$$

Lemma 2.6. [24] (Demiclosedness principle.) Let $\mathcal{C}$ be a nonempty closed convex subset of a real Hilbert space $H$. If $T: \mathcal{C} \rightarrow \mathcal{C}$ is a nonexpansive mapping with Fix $(T) \neq \emptyset$, then the mapping $I-T$ is demiclosed at 0 , i.e., if $\left\{x_{n}\right\}$ is a sequence in $\mathcal{C}$ weakly converges to $x$ and if $\left\{(I-T) x_{n}\right\}$ converges strongly to 0 , then $(I-T) x=0$.

Lemma 2.7. [27] Let $\left\{a_{n}\right\}$ be a sequence of positive real numbers such that

$$
a_{n+1} \leq\left(1-\nu_{n}\right) a_{n}+\delta_{n},
$$

where $\left\{\nu_{n}\right\}$ is a sequence in $] 0,1\left[\right.$ and $\left\{\delta_{n}\right\}$ is a sequence such that

1) $\sum_{n=1}^{\infty} \nu_{n}=\infty$;

2) $\lim _{n \rightarrow \infty} \sup \frac{\delta_{n}}{\nu_{n}} \leq 0$, or $\sum_{n=1}^{\infty}\left|\delta_{n}\right| \leq \infty$.

Hence $\lim _{n \rightarrow \infty} a_{n}=0$.

Lemma 2.8. [25] Let $\mathcal{C}$ be a nonempty closed convex subset of a real Hilbert space $\mathcal{H}$. For all $i \in$ $\{1, \ldots, N\}$, let $B_{i}$ be a strongly positive linear bounded operator on a Hilbert space $\mathcal{H}$ with coefficient $\rho_{i}>0$ and $\bar{\rho}=\min _{\{1, \ldots, N\}} \rho_{i}$. i.e., for all $x \in \mathcal{H}\left\langle B_{i} x, x\right\rangle \geq \rho_{i}\|x\|^{2}$. Moreover for each $\left.i \in\{1, \ldots, N\}, c_{i} \subset\right] 0,1\left[\right.$, with $\sum_{i=1}^{N} c_{i}=1$. Then the following properties :

(i) For all $0<\lambda<\left\|B_{i}\right\|^{-1}$, we have $\left\|I-\lambda \sum_{i=1}^{N} c_{i} B_{i}\right\| \leq 1-\lambda \bar{\rho}$, and $\left\|I-\lambda \sum_{i=1}^{N} c_{i} B_{i}\right\|$ is nonexpansive mapping.

(ii) $(\mathcal{V I \mathcal { P }})_{\left(\sum_{i=1}^{N} c_{i} B_{i}, \mathcal{C}\right)}=\bigcap_{i=1}^{N}(\mathcal{V I P})_{\left(B_{i}, \mathcal{C}\right)}$

\section{The proposed method and some properties}

In this section, we suggest and analyze our method for finding common solutions of the combination of the split equilibrium problem (2.1)-(2.2) and a finite family of nonexpansive mappings (2.3) and the combination of variational inequality problem (2.4).

Let $\mathcal{H}_{1}$ and $\mathcal{H}_{2}$ be two real Hilbert spaces. Let $\mathcal{C}$ (respectively $\mathcal{Q}$ ) be a nonempty closed convex subset of $\mathcal{H}_{1}$ (respectively $\mathcal{H}_{2}$ ). Let $F_{i}: \mathcal{C} \times \mathcal{C} \longrightarrow \mathcal{H}_{1}$ and $G_{i}: \mathcal{Q} \times \mathcal{Q} \longrightarrow \mathcal{H}_{2}$ be two finite 
family bifunctions satisfying Assumptions 2.1 such that $G_{i}$ is upper semicontinuous mapping. Let $A: \mathcal{H}_{1} \longrightarrow \mathcal{H}_{2}$ be a bounded linear operator, and let $B_{i}$ be strongly positive linear bounded operator on a Hilbert space $\mathcal{H}_{1}$ with coefficient $\rho_{i}>0$ and $\bar{\rho}=\min _{i \in\{1, \ldots, N\}} \rho_{i}$. Let $S_{n}: \mathcal{C} \longrightarrow \mathcal{C}$ be a sequence of nonexpansive mappings such that $\Gamma=\left(\cap_{n=1}^{\infty} F\left(S_{n}\right)\right) \cap \Omega \cap\left(\cap_{i=1}^{N}(\mathcal{V I P})_{B_{i}, \mathcal{C}}\right)$ and let $S: \mathcal{C} \longrightarrow \mathcal{C}$ be a mapping such that $\lim _{n \rightarrow \infty} S_{n} x=S x$.

Algorithm 3.1 For a given $x_{1} \in \mathcal{C}_{1}=\mathcal{C}$, arbitrarily, let the iterative sequences $\left\{u_{n}\right\},\left\{z_{n}\right\},\left\{y_{n}\right\}$ and $\left\{x_{n}\right\}$ be generated by

$$
\left\{\begin{array}{l}
u_{n}=T_{r_{n}}^{\sum_{i=1}^{N} a_{i} F_{i}}\left(I-\gamma A^{*}\left(I-T_{s_{n}}^{\sum_{i=1}^{N} b_{i} G_{i}}\right) A\right) x_{n} \\
z_{n}=P_{\mathcal{C}}\left[u_{n}-\lambda_{n} \sum_{i=1}^{N} c_{i} B_{i} u_{n}\right] \\
y_{n}=\alpha_{n} S_{n} x_{n}+\left(1-\alpha_{n}\right) z_{n} \\
\mathcal{C}_{n+1}=\left\{p \in \mathcal{C}_{n}:\left\|y_{n}-p\right\| \leq\left\|x_{n}-p\right\|\right\} \\
x_{n+1}=\gamma_{n} P_{\mathcal{C}_{n+1}} x_{1}+\left(1-\gamma_{n}\right) y_{n}, \quad n \geq 1
\end{array}\right.
$$

Let $\gamma \in] 0, \frac{1}{L}\left[\right.$ such that $L$ is the spectral radius of the operator $A^{*} A$, where $A^{*}$ is the adjoint of $A$. Let $\left\{s_{n}\right\}$ and $\left\{r_{n}\right\}$ be two positive real sequences, and let $\left\{\lambda_{n}\right\},\left\{\alpha_{n}\right\}$ and $\left\{\gamma_{n}\right\}$ be three sequences in $] 0,1[$, satisfying the following conditions:

C1) $0<a \leq \alpha_{n}, \gamma_{n} \leq b<1$

C2) $\sum_{i=1}^{N} a_{i}=\sum_{i=1}^{N} b_{i}=\sum_{i=1}^{N} c_{i}=1$.

C3) $\lim _{n \rightarrow \infty} \lambda_{n}=0$ and $\sum_{n=1}^{\infty}\left|\lambda_{n+1}-\lambda_{n}\right|<\infty$.

C4) $\lim _{n \rightarrow \infty} \alpha_{n}=0$ and $\sum_{n=1}^{\infty}\left|\alpha_{n+1}-\alpha_{n}\right|<\infty$.

C5) $\lim _{n \rightarrow \infty} \gamma_{n}=0, \sum_{n=1}^{\infty} \gamma_{n}=\infty$ and $\sum_{n=1}^{\infty}\left|\gamma_{n+1}-\gamma_{n}\right|<\infty$.

C6) $\lim _{n \rightarrow \infty} \inf r_{n}>0$ and $\lim _{n \rightarrow \infty} \sup s_{n}>0$.

If for $i=1,2, \ldots, N, F_{i}=F, G_{i}=G, B_{i}=B, H_{1}=H_{2}=H, S_{n}=S$ and $s_{n}=r_{n}$, then Algorithm 3.1 reduces to Algorithm 3.2 for finding the common solutions of split equilibrium problem (1.2)-(1.3), variational inequality problem (1.3) and a finite family of nonexpansive mappings (2.3).

Algorithm 3.2 For a given $x_{1} \in \mathcal{C}=\mathcal{C}$, arbitrarily, let the iterative sequences $\left\{u_{n}\right\},\left\{z_{n}\right\},\left\{y_{n}\right\}$ 
and $\left\{x_{n}\right\}$ be generated by

$$
\left\{\begin{array}{l}
u_{n}=T_{r_{n}}^{F}\left(I-\gamma A^{*}\left(I-T_{r_{n}}^{G}\right) A\right) x_{n} \\
z_{n}=P_{\mathcal{C}}\left[u_{n}-\lambda_{n} B u_{n}\right] \\
y_{n}=\alpha_{n} S x_{n}+\left(1-\alpha_{n}\right) z_{n} \\
\mathcal{C}_{n+1}=\left\{p \in \mathcal{C}_{n}:\left\|y_{n}-p\right\| \leq\left\|x_{n}-p\right\|\right\} \\
x_{n+1}=\gamma_{n} P_{\mathcal{C}_{n+1}} x_{1}+\left(1-\gamma_{n}\right) y_{n}, \quad n \geq 1
\end{array}\right.
$$

Let $\gamma \in] 0, \frac{1}{L}\left[\right.$ where $L$ is the spectral radius of the operator $A^{*} A$, and $A^{*}$ is the adjoint of $A$. Let $\left\{r_{n}\right\}$ be a positive real sequence, and let $\left\{\lambda_{n}\right\},\left\{\alpha_{n}\right\}$ and $\left\{\gamma_{n}\right\}$ be three sequences in $] 0,1[$, satisfying the following conditions:

C1) $0<a \leq \alpha_{n}, \gamma_{n} \leq b<1$.

C2) $\lim _{n \rightarrow \infty} \lambda_{n}=0$, and $\sum_{n=1}^{\infty}\left|\lambda_{n+1}-\lambda_{n}\right|<\infty$.

C3) $\lim _{n \rightarrow \infty} \alpha_{n}=0$, and $\sum_{n=1}^{\infty}\left|\alpha_{n+1}-\alpha_{n}\right|<\infty$.

C4) $\lim _{n \rightarrow \infty} \gamma_{n}=0$; and $\sum_{n=1}^{\infty} \gamma_{n}=\infty$; and $\sum_{n=1}^{\infty}\left|\gamma_{n+1}-\gamma_{n}\right|<\infty$.

C5) $\lim _{n \rightarrow \infty} \inf r_{n}>0$.

Lemma 3.1. Let $\left\{x_{n}\right\}$ be the sequence generated by the Algorithm 3.1. Then we have

(i) $\left\{x_{n}\right\}$ is well defined for every $n \in \mathbb{N}^{*}$ and bounded.

(ii) $\Gamma \subset \mathcal{C}_{n+1}$.

Proof. We show that the sequence $\left\{x_{n}\right\}$ is well defined for every $n \in \mathbb{N}^{*}$. To prove that, we will show that $\mathcal{C}_{n}$ is a closed convex subset for all $n \geq 1$. Indeed, $\mathcal{C}_{1}=\mathcal{C}$ is closed convex. Assume that $\mathcal{C}_{k}$ is closed convex; we have to prove that so is $\mathcal{C}_{k+1}$.

Let $p_{m} \in \mathcal{C}_{k+1} \subset \mathcal{C}_{k}$ such that $p_{m} \rightarrow p$ then $p \in \mathcal{C}_{k}$; (because $\mathcal{C}_{k}$ is closed) thus

$$
\left\|y_{k}-p_{m}\right\| \leq\left\|x_{k}-p_{m}\right\| \text {. }
$$

This implies that

$$
\begin{aligned}
\left\|y_{k}-p\right\| & \leq\left\|y_{k}-p_{m}\right\|+\left\|p_{m}-p\right\| \\
& \leq\left\|x_{k}-p_{m}\right\|+\left\|p_{m}-p\right\|,
\end{aligned}
$$

by taking $\lim _{m \rightarrow \infty}$ on both sides of the above estimate, hence we get

$$
\lim _{m \rightarrow \infty}\left\|y_{k}-p\right\|=\left\|y_{k}-p\right\| \leq \lim _{m \rightarrow \infty}\left(\left\|x_{k}-p_{m}\right\|+\left\|p_{m}-p\right\|\right)
$$




$$
\leq\left\|x_{k}-p\right\|
$$

Then $p \in \mathcal{C}_{k+1}$, it follows that $\mathcal{C}_{k+1}$ is closed.

Now we set $p=\lambda x+(1-\lambda) y$, for every $x, y \in \mathcal{C}_{k+1}$ then $p \in \mathcal{C}_{k}$ (because $\mathcal{C}_{k}$ is convex). From lemma 2.3, we get

$$
\begin{aligned}
\left\|y_{k}-p\right\|^{2} & =\left\|y_{k}-\lambda x-(1-\lambda) y\right\|^{2} \\
& =\left\|\lambda\left(y_{k}-x\right)+(1-\lambda)\left(y_{k}-y\right)\right\|^{2} \\
& =\lambda\left\|y_{k}-x\right\|^{2}+(1-\lambda)\left\|y_{k}-y\right\|^{2}-\lambda(1-\lambda)\|y-x\|^{2} \\
& \leq \lambda\left\|x_{k}-x\right\|^{2}+(1-\lambda)\left\|x_{k}-y\right\|^{2}-\lambda(1-\lambda)\left\|y-x_{k}+x_{k}-x\right\|^{2} \\
& =\left\|\lambda\left(x_{k}-x\right)+(1-\lambda)\left(x_{k}-y\right)\right\|^{2} \\
& =\left\|x_{k}-p\right\|^{2},
\end{aligned}
$$

thus $p \in \mathcal{C}_{k+1}$ then $\mathcal{C}_{k+1}$ is convex. Therefore $\mathcal{C}_{n}$ is closed convex for all $n \geq 1$. Since for every $x_{1} \in \mathcal{C}, P_{\mathcal{C}_{n+1}} x_{1}$ is well-defined, and $y_{n} \in \mathcal{C}_{n+1}$, then $x_{n}$ is well defined. Obviously, $\Gamma \subset \mathcal{C}_{1}$. Assume that $\Gamma \subset \mathcal{C}_{n}$, and we show that $\Gamma \subset \mathcal{C}_{n+1}$. Without loss of generality, we assume that for all $n \geq 0$, and for every $i \in\{1, \ldots, N\}, 0<\lambda_{n}<\left\|B_{i}\right\|^{-1}$. Applying Lemma 2.8, we get that $I-\lambda_{n} \sum_{i=1}^{N} c_{i} B_{i}$ is a nonexpansive mapping. Then from (1.5) for all $p \in \Gamma$, $p=P_{\mathcal{C}}\left[p-\lambda_{n} \sum_{i=1}^{N} c_{i} B_{i} p\right]$ then according to (2.7), we obtain

$$
\begin{aligned}
\left\|z_{n}-p\right\|^{2} & =\left\|P_{\mathcal{C}}\left[u_{n}-\lambda_{n} \sum_{i=1}^{N} c_{i} B_{i} u_{n}\right]-P_{\mathcal{C}}\left[p-\lambda_{n} \sum_{i=1}^{N} c_{i} B_{i} p\right]\right\|^{2} \\
& \leq\left\|u_{n}-\lambda_{n} \sum_{i=1}^{N} c_{i} B_{i} u_{n}-p+\lambda_{n} \sum_{i=1}^{N} c_{i} B_{i} p\right\|^{2} \\
& =\left\|\left(I-\lambda_{n} \sum_{i=1}^{N} c_{i} B_{i}\right) u_{n}-\left(I-\lambda_{n} \sum_{i=1}^{N} c_{i} B_{i}\right) p\right\|^{2} \\
& \leq\left\|u_{n}-p\right\|^{2} .
\end{aligned}
$$

For all $p \in \Gamma$, we have $p=T_{r_{n}}^{\sum_{i=1}^{N} a_{i} F_{i}} p$ and $\left(I-\gamma A^{*}\left(I-T_{s_{n}}^{\sum_{i=1}^{N} b_{i} G_{i}}\right) A\right) p=p$, then

$$
\begin{aligned}
\| A^{*}\left(I-T_{s_{n}}^{\sum_{i=1}^{N} b_{i} G_{i}}\right) & A x-A^{*}\left(I-T_{s_{n}}^{\sum_{i=1}^{N} b_{i} G_{i}}\right) A y \|^{2} \\
= & \left\|A^{*}\left(I-T_{s_{n}}^{\sum_{i=1}^{N} b_{i} G_{i}}\right)(A x-A y)\right\|^{2} \\
& =\left\langle A^{*}\left(I-T_{s_{n}}^{\sum_{i=1}^{N} b_{i} G_{i}}\right)(A x-A y), A^{*}\left(I-T_{s_{n}}^{\sum_{i=1}^{N} b_{i} G_{i}}\right)(A x-A y)\right\rangle \\
& =\left\langle\left(I-T_{s_{n}}^{\sum_{i=1}^{N} b_{i} G_{i}}\right)(A x-A y), A A^{*}\left(I-T_{s_{n}}^{\sum_{i=1}^{N} b_{i} G_{i}}\right)(A x-A y)\right\rangle \\
& \leq L\left\|\left(I-T_{s_{n}}^{\sum_{i=1}^{N} b_{i} G_{i}}\right)(A x-A y)\right\|^{2} .
\end{aligned}
$$


Then

$$
\left\|\left(I-T_{s_{n}}^{\sum_{i=1}^{N} b_{i} G_{i}}\right)(A x-A y)\right\|^{2} \geq \frac{1}{L}\left\|A^{*}\left(I-T_{s_{n}}^{\sum_{i=1}^{N} b_{i} G_{i}}\right)(A x-A y)\right\|^{2} .
$$

Since $T_{s_{n}}^{\sum_{i=1}^{N} b_{i} G_{i}}$ is nonexpansive, it follows from Lemma 2.1, that $I-T_{s_{n}}^{\sum_{i=1}^{N} b_{i} G_{i}}$ is 1 - inverse strongly monotone, then

$$
\begin{aligned}
\left\|\left(I-T_{s_{n}}^{\sum_{i=1}^{N} b_{i} G_{i}}\right)(A x-A y)\right\|^{2} & \leq\left\langle\left(I-T_{s_{n}}^{\sum_{i=1}^{N} b_{i} G_{i}}\right)(A x-A y),(A x-A y)\right\rangle \\
& \left.=\left\langle A^{*}\left(I-T_{s_{n}}^{\sum_{i=1}^{N} b_{i} G_{i}}\right)(A x-A y), x-y\right)\right\rangle
\end{aligned}
$$

and hence using (3.4) and (3.5), we obtain

$$
\left.\left\langle A^{*}\left(I-T_{s_{n}}^{\sum_{i=1}^{N} b_{i} G_{i}}\right)(A x-A y), x-y\right)\right\rangle \geq \frac{1}{L}\left\|A^{*}\left(I-T_{s_{n}}^{\sum_{i=1}^{N} b_{i} G_{i}}\right)(A x-A y)\right\|^{2}
$$

this implies that $A^{*}\left(I-T_{s_{n}}^{\sum_{i=1}^{N} b_{i} G_{i}}\right) A$ is $\frac{1}{L}$-inverse strongly monotone, by using Lemma 2.1 we get $I-\gamma A^{*}\left(I-T_{s_{n}}^{\sum_{i=1}^{N} b_{i} G_{i}}\right) A$ is nonexpansive for each $\left.\gamma \in\right] 0, \frac{1}{L}[$. Therefore, we obtain

$$
\begin{aligned}
\left\|u_{n}-p\right\| & =\| T_{r_{n}}^{\sum_{i=1}^{N} a_{i} F_{i}}\left(I-\gamma A^{*}\left(I-T_{s_{n}}^{\sum_{i=1}^{N} b_{i} G_{i}}\right) A\right) x_{n} \\
& -T_{r_{n}}^{\sum_{i=1}^{N} a_{i} F_{i}}\left(I-\gamma A^{*}\left(I-T_{s_{n}}^{\sum_{i=1}^{N} b_{i} G_{i}}\right) A\right) p \| \\
& \leq\left\|x_{n}-p\right\| .
\end{aligned}
$$

Let $p \in \Gamma$, then the following results can be immediately obtained from Lemma 2.3 (ii), nonexpansiveness of $S_{i},(3.3)$ and (3.6).

$$
\begin{aligned}
\left\|y_{n}-p\right\|^{2} & =\left\|\alpha_{n} S_{n} x_{n}+\left(1-\alpha_{n}\right) z_{n}-p\right\|^{2} \\
& =\left\|\alpha_{n} S_{n} x_{n}+\left(1-\alpha_{n}\right) z_{n}-\alpha_{n} S_{n} p-\left(1-\alpha_{n}\right) S_{n} p\right\|^{2} \\
& =\alpha_{n}\left\|S_{n} x_{n}-S_{n} p\right\|^{2}+\left(1-\alpha_{n}\right)\left\|z_{n}-p\right\|^{2}-\underbrace{\alpha_{n}\left(1-\alpha_{n}\right)}_{\geq 0}\left\|S_{n} x_{n}-z_{n}\right\|^{2} \\
& \leq \alpha_{n}\left\|x_{n}-p\right\|^{2}+\left(1-\alpha_{n}\right)\left\|u_{n}-p\right\|^{2} \\
& \leq \alpha_{n}\left\|x_{n}-p\right\|^{2}+\left(1-\alpha_{n}\right)\left\|x_{n}-p\right\|^{2} \\
& =\left\|x_{n}-p\right\|^{2},
\end{aligned}
$$

which yields that $p \in \mathcal{C}_{n+1}$, therefore $\Gamma \subset \mathcal{C}_{n+1}$.

Next, we show that the sequence $\left\{x_{n}\right\}$ is bounded. Note that $\left\|P_{\mathcal{C}_{n+1}} x_{1}-x_{1}\right\|^{2} \leq\left\|x^{*}-x_{1}\right\|^{2}$ for all $x^{*} \in \mathcal{C}_{n+1}$. In particular, we have

$$
\left\|P_{\mathcal{C}_{n+1}} x_{1}-x_{1}\right\|^{2} \leq\left\|P_{\Gamma} x_{1}-x_{1}\right\|^{2}
$$


Then, we get

$$
\begin{aligned}
\left\|x_{n+1}-x_{1}\right\|^{2} & =\left\|\gamma_{n}\left(P_{\mathcal{C}_{n+1}} x_{1}-x_{1}\right)+\left(1-\gamma_{n}\right)\left(y_{n}-x_{1}\right)\right\|^{2} \\
\leq & \gamma_{n}\left\|P_{\mathcal{C}_{n+1}} x_{1}-x_{1}\right\|^{2}+\left(1-\gamma_{n}\right)\left\|y_{n}-x_{1}\right\|^{2} \\
\leq & \gamma_{n}\left\|P_{\Gamma} x_{1}-x_{1}\right\|^{2}+\left(1-\gamma_{n}\right)\left\|x_{n}-x_{1}\right\|^{2}-\operatorname{see}(3.9)- \\
\leq & \max \left\{\left\|P_{\Gamma} x_{1}-x_{1}\right\|^{2},\left\|x_{n}-x_{1}\right\|^{2}\right\} \\
& \vdots \\
\leq & \max \left\{\left\|P_{\Gamma} x_{1}-x_{1}\right\|^{2}, 0\right\} \\
= & \left\|P_{\Gamma} x_{1}-x_{1}\right\|^{2} .
\end{aligned}
$$

Therefore it follows from the above inequalities that $\left\|x_{n+1}-x_{1}\right\|<\infty$, hence $\left\{x_{n}\right\}$ is bounded, and from inequalities (3.6), (3.3), and (3.9) we conclude the boundless of $\left\{u_{n}\right\},\left\{z_{n}\right\}$, and $\left\{y_{n}\right\}$.

Lemma 3.2. Let $\left\{x_{n}\right\}$ be the sequence generated by Algorithm 3.1. Then, we have
(a) $\lim _{n \rightarrow \infty}\left\|x_{n+1}-x_{n}\right\|=0$;
(b) $\lim _{n \rightarrow \infty}\left\|y_{n}-x_{n}\right\|=0$;
(c) $\lim _{n \rightarrow \infty}\left\|\left(I-T_{s_{n}}^{\sum_{i=1}^{N} b_{i} G_{i}}\right) A x_{n}\right\|=0$;
(d) $\lim _{n \rightarrow \infty}\left\|S_{n} x_{n}-z_{n}\right\|=0$;
(e) $\lim _{n \rightarrow \infty}\left\|x_{n}-z_{n}\right\|=0$.

Proof. From (2.7), we have

$$
\begin{aligned}
& \left\|z_{n}-z_{n-1}\right\| \\
& =\left\|P_{\mathcal{C}}\left[u_{n}-\lambda_{n} \sum_{i=1}^{N} c_{i} B_{i} u_{n}\right]-P_{\mathcal{C}}\left[u_{n-1}-\lambda_{n-1} \sum_{i=1}^{N} c_{i} B_{i} u_{n-1}\right]\right\| \\
& \leq\left\|\left(I-\lambda_{n} \sum_{i=1}^{N} c_{i} B_{i}\right) u_{n}-\left(I-\lambda_{n-1} \sum_{i=1}^{N} c_{i} B_{i}\right) u_{n-1}\right\| \\
& =\|\left(I-\lambda_{n} \sum_{i=1}^{N} c_{i} B_{i}\right) u_{n}-\left(I-\lambda_{n} \sum_{i=1}^{N} c_{i} B_{i}\right) u_{n-1}+\left(I-\lambda_{n} \sum_{i=1}^{N} c_{i} B_{i}\right) u_{n-1} \\
& \quad-\left(I-\lambda_{n-1} \sum_{i=1}^{N} c_{i} B_{i}\right) u_{n-1} \|
\end{aligned}
$$




$$
\leq\left\|\left(I-\lambda_{n} \sum_{i=1}^{N} c_{i} B_{i}\right) u_{n}-\left(I-\lambda_{n} \sum_{i=1}^{N} c_{i} B_{i}\right) u_{n-1}\right\|+\left\|\left(\lambda_{n-1}-\lambda_{n}\right) \sum_{i=1}^{N} c_{i} B_{i} u_{n-1}\right\| .
$$

According to Lemma 2.8, we obtain

$$
\left\|z_{n}-z_{n-1}\right\| \leq\left\|u_{n}-u_{n-1}\right\|+\left|\lambda_{n}-\lambda_{n-1}\right|\left\|\sum_{i=1}^{N} c_{i} B_{i} u_{n-1}\right\| .
$$

Next, we apply the definition of $y_{n}$, and the inequality (3.10), we get

$$
\begin{aligned}
& \left\|y_{n}-y_{n-1}\right\| \\
& =\left\|\alpha_{n} S_{n} x_{n}+\left(1-\alpha_{n}\right) z_{n}-\alpha_{n-1} S_{n-1} x_{n-1}-\left(1-\alpha_{n-1}\right) z_{n-1}\right\| \\
& =\| \alpha_{n}\left(S_{n} x_{n}-S_{n} x_{n-1}\right)+\left(\alpha_{n}-\alpha_{n-1}\right) S_{n} x_{n-1}+\alpha_{n-1}\left(S_{n} x_{n-1}-S_{n-1} x_{n-1}\right) \\
& +\left(1-\alpha_{n}\right)\left(z_{n}-z_{n-1}\right)+\left(\alpha_{n-1}-\alpha_{n}\right) z_{n-1} \| \\
& \leq \alpha_{n}\left\|x_{n}-x_{n-1}\right\|+\left|\alpha_{n}-\alpha_{n-1}\right|\left(\left\|S_{n} x_{n-1}\right\|+\left\|z_{n-1}\right\|\right) \\
& +\alpha_{n-1}\left\|S_{n} x_{n-1}-S_{n-1} x_{n-1}\right\|+\left(1-\alpha_{n}\right)\left\|z_{n}-z_{n-1}\right\| \\
& \leq \alpha_{n}\left\|x_{n}-x_{n-1}\right\|+\left|\alpha_{n}-\alpha_{n-1}\right|\left(\left\|S_{n} x_{n-1}\right\|+\left\|z_{n-1}\right\|\right)+\alpha_{n-1}\left\|S_{n} x_{n-1}-S_{n-1} x_{n-1}\right\| \\
& +\left(1-\alpha_{n}\right)\left(\left\|u_{n}-u_{n-1}\right\|+\left|\lambda_{n}-\lambda_{n-1}\right|\left\|\sum_{i=1}^{N} c_{i} B_{i} u_{n-1}\right\|\right) \text {. }
\end{aligned}
$$

Using the above inequalities, we obtain the following result, for all $n \geq 1$,

$$
\begin{aligned}
\| x_{n+1}- & x_{n} \| \\
= & \| \gamma_{n}\left(P_{\mathcal{C}_{n+1}} x_{1}-P_{\mathcal{C}_{n}} x_{1}\right)+\left(\gamma_{n}-\gamma_{n-1}\right) P_{\mathcal{C}_{n}} x_{1}+\gamma_{n}\left(y_{n-1}-y_{n}\right)+\left(\gamma_{n-1}-\gamma_{n}\right) y_{n-1} \\
& \quad+y_{n}-y_{n-1} \| \\
\leq & \left\|P_{\mathcal{C}_{n+1}} x_{1}-P_{\mathcal{C}_{n}} x_{1}\right\|+\left|\gamma_{n}-\gamma_{n-1}\right|\left(\left\|P_{\mathcal{C}_{n}} x_{1}\right\|+\left\|y_{n-1}\right\|\right)+\left(1-\gamma_{n}\right)\left\|y_{n}-y_{n-1}\right\| \\
\leq & \left\|P_{\mathcal{C}_{n+1}} x_{1}-P_{\mathcal{C}_{n}} x_{1}\right\|+\left|\gamma_{n}-\gamma_{n-1}\right|\left(\left\|P_{\mathcal{C}_{n}} x_{1}\right\|+\left\|y_{n-1}\right\|\right) \\
& +\left(1-\gamma_{n}\right)\left(\alpha_{n}\left\|x_{n}-x_{n-1}\right\|+\left|\alpha_{n}-\alpha_{n-1}\right|\left(\left\|S_{n} x_{n-1}\right\|+\left\|z_{n-1}\right\|\right)+\right. \\
& \quad+\left|\alpha_{n}-\alpha_{n-1}\right|\left(\left\|S_{n} x_{n-1}\right\|+\left\|z_{n-1}\right\|\right)+\alpha_{n-1}\left(\left\|S_{n} x_{n-1}\right\|+\left\|S_{n-1} x_{n-1}\right\|\right) \\
& \quad+\left(1-\alpha_{n}\right)\left(\left\|u_{n}\right\|+\left\|u_{n-1}\right\|+\left|\lambda_{n}-\lambda_{n-1}\right|\left\|\sum_{i=1}^{N} c_{i} B_{i} u_{n-1}\right\|\right) \\
& \quad\left(1-\gamma_{n-1}\right)\left\|x_{n}-x_{n-1}\right\|+\left\|P_{\mathcal{C}_{n+1}} x_{1}-P_{\mathcal{C}_{n}} x_{1}\right\|+\left|\gamma_{n}-\gamma_{n-1}\right|\left(\left\|P_{\mathcal{C}_{n}} x_{1}\right\|+\left\|y_{n-1}\right\|\right)
\end{aligned}
$$




$$
\begin{aligned}
& \leq\left(1-\gamma_{n}\right)\left\|x_{n}-x_{n-1}\right\|+M\left(1+\left|\gamma_{n}-\gamma_{n-1}\right|+\left|\alpha_{n}-\alpha_{n-1}\right|+\alpha_{n-1}-\alpha_{n}+1\right. \\
& \left.\quad+\left|\lambda_{n}-\lambda_{n-1}\right|\right) .
\end{aligned}
$$

where

$$
\begin{aligned}
M=\max \{ & \sup _{n \geq 1}\left(\left\|P_{\mathcal{C}_{n+1}} x_{1}\right\|+\left\|P_{\mathcal{C}_{n}} x_{1}\right\|\right), \sup _{n \geq 1}\left(\left\|P_{\mathcal{C}_{n}} x_{1}\right\|+\left\|y_{n-1}\right\|\right), \\
& \sup _{n \geq 1}\left(\left\|S_{n} x_{n-1}\right\|-\left\|z_{n-1}\right\|\right), \sup _{n \geq 1}\left(\left\|S_{n} x_{n-1}\right\|+\left\|S_{n-1} x_{n-1}\right\|\right), \\
& \left.\sup _{n \geq 1}\left(\left\|u_{n}\right\|+\left\|u_{n-1}\right\|\right), \sup _{n \geq 1}\left(\left\|\sum_{i=1}^{N} c_{i} B_{i} u_{n-1}\right\|\right)\right\} .
\end{aligned}
$$

Setting $\delta_{n}=M\left(2+\left|\gamma_{n}-\gamma_{n-1}\right|+2\left|\alpha_{n}-\alpha_{n-1}\right|+\left|\lambda_{n}-\lambda_{n-1}\right|\right)$ and by using conditions C3), C4) and C5), it follows that $\sum_{n=1}^{\infty} \delta_{n}<\infty$ and $\sum_{n=1}^{\infty} \gamma_{n}=\infty$. Hence from Lemma 2.7 we conclude directly that $\lim _{n \rightarrow \infty}\left\|x_{n+1}-x_{n}\right\|=0$ which proves the result (a).

Now, we show the assertion (b). We have

$$
\begin{aligned}
\left\|y_{n}-x_{n}\right\| & \leq\left\|y_{n}-x_{n+1}\right\|+\left\|x_{n+1}-x_{n}\right\| \\
& =\left\|y_{n}-\gamma_{n} P_{\mathcal{C}_{n+1}} x_{1}-\left(1-\gamma_{n}\right) y_{n}\right\|+\left\|x_{n+1}-x_{n}\right\| \\
& =\left\|\gamma_{n}\left(y_{n}-P_{\mathcal{C}_{n+1}} x_{1}\right)\right\|+\left\|x_{n+1}-x_{n}\right\| \\
& \leq \gamma_{n}\left\|y_{n}-P_{\mathcal{C}_{n+1}} x_{1}\right\|+\left\|x_{n+1}-x_{n}\right\| \\
& \leq \gamma_{n}\left\|x_{n}-P_{\mathcal{C}_{n+1}} x_{1}\right\|+\left\|x_{n+1}-x_{n}\right\| \\
& =\gamma_{n}\left\|x_{n}-P_{\Gamma} x_{1}\right\|+\left\|x_{n+1}-x_{n}\right\| \\
& \leq \gamma_{n}\left(\left\|x_{n}\right\|+\left\|P_{\Gamma} x_{1}\right\|\right)+\left\|x_{n+1}-x_{n}\right\|
\end{aligned}
$$

This implies with C5) condition and (a), $\lim _{n \rightarrow \infty}\left\|y_{n}-x_{n}\right\|=0$, thus (b) is proved.

Now we prove the assertion (c). For $p \in \Gamma$, we have $p=T_{r_{n}}^{\sum_{i=1}^{N} a_{i} F_{i}} p$. Using Lemma 2.4 we get

$$
\begin{aligned}
& \left\|u_{n}-p\right\|^{2} \\
& \quad=\left\|T_{r_{n}}^{\sum_{i=1}^{N} a_{i} F_{i}}\left(I-\gamma A^{*}\left(I-T_{s_{n}}^{\sum_{i=1}^{N} b_{i} G_{i}}\right) A\right) x_{n}-T_{r_{n}}^{\sum_{i=1}^{N} a_{i} F_{i}} p\right\|^{2} \\
& \quad \leq\left\|x_{n}-p-\gamma A^{*}\left(I-T_{s_{n}}^{\sum_{i=1}^{N} b_{i} G_{i}}\right) A x_{n}\right\|^{2} \\
& \quad=\left\|x_{n}-p\right\|^{2}+\gamma^{2}\left\|A^{*}\left(I-T_{s_{n}}^{\sum_{i=1}^{N} b_{i} G_{i}}\right) A x_{n}\right\|^{2}-2 \gamma\left\langle x_{n}-p, A^{*}\left(I-T_{s_{n}}^{\sum_{i=1}^{N} b_{i} G_{i}}\right) A x_{n}\right\rangle \\
& \quad=\left\|x_{n}-p\right\|^{2}+\gamma^{2}\left\langle A^{*}\left(I-T_{s_{n}}^{\sum_{i=1}^{N} b_{i} G_{i}}\right) A x_{n}, A^{*}\left(I-T_{s_{n}}^{\sum_{i=1}^{N} b_{i} G_{i}}\right) A x_{n}\right\rangle \\
& \quad-2 \gamma\left\langle A\left(x_{n}-p\right),\left(I-T_{s_{n}}^{\sum_{i=1}^{N} b_{i} G_{i}}\right) A x_{n}\right\rangle
\end{aligned}
$$




$$
\begin{aligned}
& \leq\left\|x_{n}-p\right\|^{2}+\gamma^{2} L\left\|\left(I-T_{s_{n}}^{\sum_{i=1}^{N} b_{i} G_{i}}\right) A x_{n}\right\|^{2} \\
& -2 \gamma\left\langle A\left(x_{n}-p\right)+\left(I-T_{s_{n}}^{\sum_{i=1}^{N} b_{i} G_{i}}\right) A x_{n}-\left(I-T_{s_{n}}^{\sum_{i=1}^{N} b_{i} G_{i}}\right) A x_{n},\left(I-T_{s_{n}}^{\sum_{i=1}^{N} b_{i} G_{i}}\right) A x_{n}\right\rangle \\
& =\left\|x_{n}-p\right\|^{2}+\gamma^{2} L\left\|\left(I-T_{s_{n}}^{\sum_{i=1}^{N} b_{i} G_{i}}\right) A x_{n}\right\|^{2}-2 \gamma\left\|\left(I-T_{s_{n}}^{\sum_{i=1}^{N} b_{i} G_{i}}\right) A x_{n}\right\|^{2} \\
& -2 \gamma\left\langle T_{s_{n}{ }_{i=1}^{N} b_{i} G_{i}} A x_{n}-A p,\left(I-T_{s_{n}}^{\sum_{i=1}^{N} b_{i} G_{i}}\right) A x_{n}\right\rangle .
\end{aligned}
$$

It follows from (2.10) that

$$
\begin{aligned}
\left\|u_{n}-p\right\|^{2} & \leq\left\|x_{n}-p\right\|^{2}+\gamma^{2} L\left\|\left(I-T_{s_{n}}^{\sum_{i=1}^{N} b_{i} G_{i}}\right) A x_{n}\right\|^{2}-2 \gamma\left\|\left(I-T_{s_{n}}^{\sum_{i=1}^{N} b_{i} G_{i}}\right) A x_{n}\right\|^{2} \\
& +2 \gamma \frac{1}{2}\left\|\left(I-T_{s_{n}}^{\sum_{i=1}^{N} b_{i} G_{i}}\right) A x_{n}\right\|^{2} \\
& =\left\|x_{n}-p\right\|^{2}+\gamma(\gamma L-1)\left\|\left(I-T_{s_{n}}^{\sum_{i=1}^{N} b_{i} G_{i}}\right) A x_{n}\right\|^{2} .
\end{aligned}
$$

Since

$$
\begin{aligned}
\| y_{n} & -p \|^{2} \\
& =\left\|\alpha_{n} S_{n} x_{n}+\left(1-\alpha_{n}\right) z_{n}-p\right\|^{2} \\
& =\left\|\alpha_{n}\left(S_{n} x_{n}-S_{n} p\right)+\left(1-\alpha_{n}\right)\left(z_{n}-p\right)\right\|^{2} \\
& =\alpha_{n}\left\|S_{n} x_{n}-S_{n} p\right\|^{2}+\left(1-\alpha_{n}\right)\left\|z_{n}-p\right\|^{2}-\alpha_{n}\left(1-\alpha_{n}\right)\left\|S_{n} x_{n}-z_{n}\right\|^{2} \\
& \leq \alpha_{n}\left\|x_{n}-p\right\|^{2}+\left(1-\alpha_{n}\right)\left\|u_{n}-p\right\|^{2} \\
& \leq \alpha_{n}\left\|x_{n}-p\right\|^{2}+\left(1-\alpha_{n}\right)\left(\left\|x_{n}-p\right\|^{2}+\gamma(\gamma L-1)\left\|\left(I-T_{s_{n}}^{\sum_{i=1}^{N} b_{i} G_{i}}\right) A x_{n}\right\|^{2}\right) \\
& =\left\|x_{n}-p\right\|^{2}+\left(1-\alpha_{n}\right) \gamma(\gamma L-1)\left\|\left(I-T_{s_{n}}^{\sum_{i=1}^{N} b_{i} G_{i}}\right) A x_{n}\right\|^{2} .
\end{aligned}
$$

Hence

$$
\begin{aligned}
\left(1-\alpha_{n}\right) \gamma(1-\gamma L)\left\|\left(I-T_{s_{n}}^{\sum_{i=1}^{N} b_{i} G_{i}}\right) A x_{n}\right\|^{2} & \leq\left\|x_{n}-p\right\|^{2}-\left\|y_{n}-p\right\|^{2} \\
& =\left\|x_{n}-y_{n}\right\|\left(\left\|x_{n}-p\right\|+\left\|y_{n}-p\right\|\right) .
\end{aligned}
$$

Using (b), condition $\mathbf{C 1}$ ) and letting $n \longrightarrow \infty$, we obtain the desired result.

In the following, we show the assertion (d); let $p \in \Gamma$, from (3.7), (3.3) and (3.6), we get

$$
\begin{aligned}
\left\|y_{n}-p\right\|^{2} & \leq \alpha_{n}\left\|x_{n}-p\right\|^{2}+\left(1-\alpha_{n}\right)\left\|z_{n}-p\right\|^{2}-\alpha_{n}\left(1-\alpha_{n}\right)\left\|S_{n} x_{n}-z_{n}\right\|^{2} \\
& \leq\left\|x_{n}-p\right\|^{2}-\alpha_{n}\left(1-\alpha_{n}\right)\left\|S_{n} x_{n}-z_{n}\right\|^{2}
\end{aligned}
$$

\section{Consequently}

$$
\begin{aligned}
\alpha_{n}\left(1-\alpha_{n}\right)\left\|S_{n} x_{n}-z_{n}\right\|^{2} & \leq\left\|x_{n}-p\right\|^{2}-\left\|y_{n}-p\right\|^{2} \\
& =\left(\left\|x_{n}-p\right\|+\left\|y_{n}-p\right\|\right)\left\|x_{n}-y_{n}\right\| .
\end{aligned}
$$


From the condition $\mathbf{C 1}$ ), it holds that

$$
a(1-b)\left\|S_{n} x_{n}-z_{n}\right\|^{2} \leq\left(\left\|x_{n}-p\right\|+\left\|y_{n}-p\right\|\right)\left\|x_{n}-y_{n}\right\| .
$$

By using (b), it can be easily seen that $\lim _{n \rightarrow \infty}\left\|S_{n} x_{n}-z_{n}\right\|=0$.

Next, we show the assertion (e), we have

$$
\left\|y_{n}-z_{n}\right\|=\alpha_{n}\left\|S_{n} x_{n}-z_{n}\right\| .
$$

Furthermore

$$
\begin{aligned}
\left\|z_{n}-x_{n}\right\| & \leq\left\|z_{n}-x_{n+1}\right\|+\left\|x_{n+1}-x_{n}\right\| \\
& =\left\|z_{n}-\gamma_{n} P_{\mathcal{C}_{n+1}} x_{1}-\left(1-\gamma_{n}\right) y_{n}\right\|+\left\|x_{n+1}-x_{n}\right\| \\
& \leq \gamma_{n}\left\|z_{n}-P_{\mathcal{C}_{n+1}} x_{1}\right\|+\left(1-\gamma_{n}\right)\left\|z_{n}-y_{n}\right\|+\left\|x_{n+1}-x_{n}\right\| \\
& \leq \gamma_{n}\left(\left\|P_{\mathcal{C}_{n+1}} x_{1}\right\|+\left\|z_{n}\right\|\right)+\left(1-\gamma_{n}\right)\left\|y_{n}-z_{n}\right\|+\left\|x_{n+1}-x_{n}\right\| .
\end{aligned}
$$

Combining the above inequality with (3.13), we obtain

$\left\|z_{n}-x_{n}\right\| \leq \gamma_{n}\left(\left\|P_{\mathcal{C}_{n+1}} x_{1}\right\|+\left\|z_{n}\right\|\right)+\left(1-\gamma_{n}\right) \alpha_{n}\left\|S_{n} x_{n}-z_{n}\right\|+\left\|x_{n+1}-x_{n}\right\|$.

We apply (d), (a) and the conditions C4) and C5) of Algorithm 3.1, so we get immediately that $\lim _{n \rightarrow \infty}\left\|z_{n}-x_{n}\right\|=0$.

Theorem 3.1. The sequence $\left\{x_{n}\right\}$ generated by Algorithm 3.1 converges strongly to $q \in \Gamma$, where $\Gamma=\left(\cap_{n=1}^{\infty} F\left(S_{n}\right)\right) \cap \Omega \cap\left(\cap_{i=1}^{N}(\mathcal{V I P})_{B_{i}, \mathcal{C}}\right)$, and

$\Omega=\left\{p \in \mathcal{C}: p \in \cap_{i=1}^{N} E P\left(F_{i}\right)\right.$ and $A p \in \cap_{i=1}^{N} E P\left(G_{i}\right)$, for all $\left.i \in\{1, \ldots, N\}\right\}$.

Proof. Firstly, we show that $q \in \cap_{n=1}^{\infty} F\left(S_{n}\right)$. We know that $x_{n}$ is bounded, then there exist a subsequence $x_{n_{j}}$ such that $x_{n_{j}} \rightarrow q$.

Since $\left\|S_{n_{j}} x_{n_{j}}-x_{n_{j}}\right\| \leq\left\|S_{n} x_{n_{j}}-z_{n_{j}}\right\|+\left\|z_{n_{j}}-x_{n_{j}}\right\|$. From (d) and (e) of Lemma 3.2, we get $\lim _{j \rightarrow \infty}\left\|S_{n_{j}} x_{n_{j}}-x_{n_{j}}\right\|=0$. Using Lemma 2.6, we get $S_{n} q-q=0$, then $q \in F\left(S_{n}\right)$ for every $n \in \mathbb{N}^{*}$. Hence $q \in \cap_{n=1}^{\infty} F\left(S_{n}\right)$.

Next, we show that $q \in \Omega=\left\{p \in \mathcal{C}: p \in \cap_{i=1}^{N} E P\left(F_{i}\right)\right.$ and $\left.A p \in \cap_{i=1}^{N} E P\left(G_{i}\right)\right\}$. Let us first prove that $\lim _{n \rightarrow \infty}\left\|u_{n}-x_{n}\right\|=0$. For a given $p \in \Gamma$, we easily obtain

$$
\begin{aligned}
\| u_{n}-p & \|^{2} \\
& =\left\|T_{r_{n}}^{\sum_{i=1}^{N} a_{i} F_{i}}\left(x_{n}-\gamma A^{*}\left(I-T_{s_{n}}^{\sum_{i=1}^{N} b_{i} G_{i}}\right) A x_{n}\right)-T_{r_{n}}^{\sum_{i=1}^{N} a_{i} F_{i}} p\right\|^{2} \\
& \leq\left\langle T_{r_{n}}^{\sum_{i=1}^{N} a_{i} F_{i}}\left(x_{n}-\gamma A^{*}\left(I-T_{s_{n}}^{\sum_{i=1}^{N} b_{i} G_{i}}\right) A x_{n}\right)\right.
\end{aligned}
$$




$$
\begin{aligned}
& \left.-T_{r_{n}}^{\sum_{i=1}^{N} a_{i} F_{i}} p, x_{n}-p-\gamma A^{*}\left(I-T_{s_{n}}^{\sum_{i=1}^{N} b_{i} G_{i}}\right) A x_{n}\right\rangle \\
& =\left\langle u_{n}-p, x_{n}-p-\gamma A^{*}\left(I-T_{s_{n}}^{\sum_{i=1}^{N} b_{i} G_{i}}\right) A x_{n}\right\rangle .
\end{aligned}
$$

From Lemma 2.3, it follows that

$$
\begin{aligned}
\| u_{n}-p & \|^{2} \\
\leq & \frac{1}{2}\left(\left\|u_{n}-p\right\|^{2}+\left\|x_{n}-p-\gamma A^{*}\left(I-T_{s_{n}}^{\sum_{i=1}^{N} b_{i} G_{i}}\right) A x_{n}\right\|^{2}\right. \\
& \left.\quad-\left\|u_{n}-x_{n}+\gamma A^{*}\left(I-T_{s_{n}}^{\sum_{i=1}^{N} b_{i} G_{i}}\right) A x_{n}\right\|^{2}\right) \\
\leq & \frac{1}{2}\left(\left\|u_{n}-p\right\|^{2}+\left\|x_{n}-p\right\|^{2}-2 \gamma\left\langle A^{*}\left(I-T_{s_{n}}^{\sum_{i=1}^{N} b_{i} G_{i}}\right) A x_{n}, x_{n}-p\right\rangle\right. \\
& \quad+\gamma^{2}\left\|A^{*}\left(I-T_{s_{n}}^{\sum_{i=1}^{N} b_{i} G_{i}}\right) A x_{n}\right\|^{2}-\left\|u_{n}-x_{n}\right\|^{2} \\
& \left.\quad-2 \gamma\left\langle A^{*}\left(I-T_{s_{n}}^{\sum_{i=1}^{N} b_{i} G_{i}}\right) A x_{n}, u_{n}-x_{n}\right\rangle-\gamma^{2}\left\|A^{*}\left(I-T_{s_{n}}^{\sum_{i=1}^{N} b_{i} G_{i}}\right) A x_{n}\right\|^{2}\right) .
\end{aligned}
$$

Then

$$
\left\|u_{n}-p\right\|^{2} \leq\left\|x_{n}-p\right\|^{2}-\left\|u_{n}-x_{n}\right\|^{2}-2 \gamma\left\langle A^{*}\left(I-T_{s_{n}}^{\sum_{i=1}^{N} b_{i} G_{i}}\right) A x_{n}, u_{n}-p\right\rangle .
$$

Substituting (3.8) into (3.14), we obtain

$$
\begin{gathered}
\left\|y_{n}-p\right\|^{2} \leq \alpha_{n}\left\|x_{n}-p\right\|^{2}+\left(1-\alpha_{n}\right)\left(\left\|x_{n}-p\right\|^{2}-\left\|u_{n}-x_{n}\right\|^{2}\right. \\
\left.-2 \gamma\left\langle A^{*}\left(I-T_{s_{n}}^{\sum_{i=1}^{N} b_{i} G_{i}}\right) A x_{n}, u_{n}-p\right\rangle\right) \\
=\left\|x_{n}-p\right\|^{2}-\left(1-\alpha_{n}\right)\left\|u_{n}-x_{n}\right\|^{2}-2 \gamma\left(1-\alpha_{n}\right)\left\langle A^{*}\left(I-T_{s_{n}}^{\sum_{i=1}^{N} b_{i} G_{i}}\right) A x_{n}, u_{n}-p\right\rangle .
\end{gathered}
$$

Therefore

$$
\begin{aligned}
& \left(1-\alpha_{n}\right)\left\|u_{n}-x_{n}\right\|^{2} \\
& \quad \leq\left\|x_{n}-p\right\|^{2}-\left\|y_{n}-p\right\|^{2}-2 \gamma\left(1-\alpha_{n}\right)\left\langle A^{*}\left(I-T_{s_{n}}^{\sum_{i=1}^{N} b_{i} G_{i}}\right) A x_{n}, u_{n}-p\right\rangle \\
& \quad \leq\left(\left\|x_{n}-p\right\|+\left\|y_{n}-p\right\|\right)\left\|y_{n}-x_{n}\right\| \\
& \quad+2 \gamma\left(1-\alpha_{n}\right)\left\|\left(I-T_{s_{n}}^{\sum_{i=1}^{N} b_{i} G_{i}}\right) A x_{n}\right\|\left\|A\left(u_{n}-p\right)\right\| .
\end{aligned}
$$

Letting $n \longrightarrow \infty$ and using (b), (c) and both conditions C1) and C2) of Algorithm 3.1, we get

$$
\lim _{n \rightarrow \infty}\left\|u_{n}-x_{n}\right\|=0
$$

Now, we are ready to prove that for every $i \in\{1, \ldots, N\}$, we have $p \in \cap_{i=1}^{N} E P\left(F_{i}\right)$. Indeed; for all $i \in\{1, \ldots, N\}$, we have from the definition of $u_{n}$

$$
u_{n}=T_{r_{n}}^{\sum_{i=1}^{N} a_{i} F_{i}}\left(I-\gamma A^{*}\left(I-T_{s_{n}}^{\sum_{i=1}^{N} b_{i} G_{i}}\right) A\right) x_{n}, \quad n \geq 1 .
$$


Then

$$
\sum_{i=1}^{N} a_{i} F_{i}\left(u_{n}, y\right)+\frac{1}{r_{n}}\left\langle y-u_{n}, u_{n}-x_{n}+\gamma A^{*}\left(I-T_{s_{n}}^{\sum_{i=1}^{N} b_{i} G_{i}}\right) A x_{n}\right\rangle \geq 0 \quad \forall y \in \mathcal{C},
$$

which implies

$$
-\sum_{i=1}^{N} a_{i} F_{i}\left(u_{n}, y\right) \leq \frac{1}{r_{n}}\left(\left\langle y-u_{n}, u_{n}-x_{n}\right\rangle+\gamma\left\langle y-u_{n}, A^{*}\left(I-T_{s_{n}}^{\sum_{i=1}^{N} b_{i} G_{i}}\right) A x_{n}\right\rangle\right) .
$$

By monotonicity of $F_{i}$ and from Lemma 2.5, we can write the above inequality as follows

$$
\sum_{i=1}^{N} a_{i} F_{i}\left(y, u_{n}\right) \leq \frac{1}{r_{n}}\left(\left\langle y-u_{n}, u_{n}-x_{n}\right\rangle+\gamma\left\langle y-u_{n}, A^{*}\left(I-T_{s_{n}}^{\sum_{i=1}^{N} b_{i} G_{i}}\right) A x_{n}\right\rangle\right) .
$$

Therefore

$\sum_{i=1}^{N} a_{i} F_{i}\left(y, u_{n_{j}}\right) \leq \frac{1}{r_{n_{j}}}\left(\left\|y-u_{n_{j}}\right\|\left\|u_{n_{j}}-x_{n_{j}}\right\|+\gamma\left\|A\left(y-u_{n_{j}}\right)\right\|\left\|\left(I-T_{s_{n_{j}}}^{\sum_{i=1}^{N} b_{i} G_{i}}\right) A x_{n_{j}}\right\|\right)$.

From Lemma 3.2, (3.15) and the condition C6) of Algorithm 3.1, we can conclude that for every $i \in\{1, \ldots, N\}, \lim _{j \rightarrow \infty} \sum_{i=1}^{N} a_{i} F_{i}\left(y, u_{n_{j}}\right) \leq 0$, and since $\sum_{i=1}^{N} a_{i} F_{i}$ is lower semicontinuous in the last argument (see Lemma 2.5), hence we get for each $i \in\{1, \ldots, N\}$, and for every $y \in \mathcal{C}$

$$
\sum_{i=1}^{N} a_{i} F_{i}(y, q) \leq 0 .
$$

Setting $y_{t}=t y+(1-t) q$ for some $0<t \leq 1$, then $y_{t} \in \mathcal{C}$. Using the assertions 1 and 4 of Assumption 2.1, we get

$$
\begin{aligned}
0 & =\sum_{i=1}^{N} a_{i} F_{i}\left(y_{t}, y_{t}\right) \\
& =\sum_{i=1}^{N} a_{i} F_{i}\left(y_{t}, t y+(1-t) q\right) \\
& \leq t \sum_{i=1}^{N} a_{i} F_{i}\left(y_{t}, y\right)+\underbrace{(1-t) \sum_{i=1}^{N} a_{i} F_{i}\left(y_{t}, q\right)}_{\leq 0} \\
& \leq t \sum_{i=1}^{N} a_{i} F_{i}\left(y_{t}, y\right)
\end{aligned}
$$

Then for all $i \in\{1, \ldots, N\}, \sum_{i=1}^{N} a_{i} F_{i}(t y+(1-t) q, y) \geq 0$, letting $t \longrightarrow 0$, by the hemicontinuity of $\sum_{i=1}^{N} a_{i} F_{i}$ (see Lemma 2.5), we obtain that $\sum_{i=1}^{N} a_{i} F_{i}(q, y) \geq 0$, for each $i \in\{1, \ldots, N\}$. 
Then $q \in E P\left(\sum_{i=1}^{N} a_{i} F_{i}\right)$ for every $i \in\{1, \ldots, N\}$. According to Lemma 2.5, we easily deduce that $q \in \cap_{i=1}^{N} E P\left(F_{i}\right)$.

Next, we show that for all $i \in\{1, \ldots, N\}, A q \in \cap_{i=1}^{N} E P\left(G_{i}\right)$.

For all $i \in\{1, \ldots, N\}$, we have $A$ is bounded and since $x_{n_{j}} \rightarrow q$ then $A x_{n_{j}} \rightarrow A q$, from Lemma 3.2 (c), we get $T_{s_{n_{j}, i}}^{\sum_{i=1}^{N} b_{i} G_{i}} A x_{n_{j}} \rightarrow A q$, thus

$G_{i}\left(T_{s_{n_{j}}}^{\sum_{i=1}^{N} b_{i} G_{i}} A x_{n_{j}}, z\right)+\frac{1}{s_{n_{j}}}\left\langle z-T_{s_{n_{j}}}^{\sum_{i=1}^{N} b_{i} G_{i}} A x_{n_{j}}, T_{s_{n_{j}, i}}^{\sum_{i=1}^{N} b_{i} G_{i}} A x_{n_{j}}-A x_{n_{j}}\right\rangle \geq 0 \quad \forall z \in \mathcal{C}$.

Taking the limit sup of each side of the above inequality, and using the fact that $G_{i}$ is upper semicontinuous in the first argument and using the condition $C 5$ ), we conclude that for every $i \in\{1, \ldots N\}$,

$$
G_{i}(A q, z) \geq 0, \quad \forall z \in \mathcal{C}
$$

which implies that $A q \in E P\left(G_{i}\right)$, then $A q \in \cap_{i=1}^{N} E P\left(G_{i}\right)$ and hence $q \in \Omega$.

Furthermore, we show that $q \in \cap_{i=1}^{N}(\mathcal{V I P})_{B_{i}, \mathcal{C}}$. Let

$$
T v=\left\{\begin{array}{c}
\sum_{i=1}^{N} c_{i} B_{i} v+N_{\mathcal{C}} v, \quad \forall v \in \mathcal{C}, \\
\emptyset \quad \text { otherwise }
\end{array}\right.
$$

where $N_{C} v:=\{w \in H:\langle w, v-u\rangle \geq 0, \quad \forall u \in C\}$ is the normal cone to $C$ at $v \in C$. Then $T$ is maximal monotone and $0 \in T v$ if and only if $v \in(\mathcal{V I P})_{\sum_{i=1}^{N} c_{i} B_{i}, \mathcal{C}}$ (see [23]).

Let $G(T)$ denote the graph of $T$, and let $(v, u) \in G(T)$; since $u-\sum_{i=1}^{N} c_{i} B_{i} \in N_{\mathcal{C}} v$, and $z_{n} \in \mathcal{C}$, we have

$$
\left\langle v-z_{n}, u-\sum_{i=1}^{N} c_{i} B_{i} v\right\rangle \geq 0
$$

It follows from $z_{n}=P_{C}\left[u_{n}-\lambda_{n} \sum_{i=1}^{N} c_{i} B_{i} u_{n}\right]$ and $v \in C$ that

$$
\left\langle v-z_{n}, z_{n}-\left(u_{n}-\lambda_{n} \sum_{i=1}^{N} c_{i} B_{i} u_{n}\right)\right\rangle \geq 0
$$

Then in particular, it follows that

$$
\left\langle v-z_{n_{k}}, \frac{z_{n_{k}}-u_{n_{k}}}{\lambda_{n_{k}}}+\sum_{i=1}^{N} c_{i} B_{i} u_{n_{k}}\right\rangle \geq 0 .
$$

Using the fact that $\sum_{i=1}^{N} c_{i} B_{i}$ is strongly positive, (3.17), and (3.18), we obtain

$$
\left\langle v-z_{n_{k}}, u\right\rangle \geq\left\langle v-z_{n_{k}}, \sum_{i=1}^{N} c_{i} B_{i} v\right\rangle
$$




$$
\begin{aligned}
& \geq\left\langle v-z_{n_{k}}, \sum_{i=1}^{N} c_{i} B_{i} v\right\rangle-\left\langle v-z_{n_{k}}, \frac{z_{n_{k}}-u_{n_{k}}}{\lambda_{n_{k}}}+\sum_{i=1}^{N} c_{i} B_{i} u_{n_{k}}\right\rangle \\
& =\underbrace{\left\langle v-z_{n_{k}}, \sum_{i=1}^{N} c_{i} B_{i}\left(v-z_{n_{k}}\right)\right\rangle}_{\geq 0}-\left\langle v-z_{n_{k}}, \frac{z_{n_{k}}-u_{n_{k}}}{\lambda_{n_{k}}}\right\rangle \\
& +\left\langle v-z_{n_{k}}, \sum_{i=1}^{N} c_{i} B_{i} z_{n_{k}}-\sum_{i=1}^{N} c_{i} B_{i} u_{n_{k}}\right\rangle \\
& \geq-\left\langle v-z_{n_{k}}, \frac{z_{n_{k}}-u_{n_{k}}}{\lambda_{n_{k}}}\right\rangle+\left\langle v-z_{n_{k}}, \sum_{i=1}^{N} c_{i} B_{i} z_{n_{k}}-\sum_{i=1}^{N} c_{i} B_{i} u_{n_{k}}\right\rangle .
\end{aligned}
$$

Note that

$$
\left\|z_{n}-u_{n}\right\| \leq\left\|z_{n}-x_{n}\right\|+\left\|x_{n}-u_{n}\right\|,
$$

it follows from Lemma 3.2 (e) and (3.15) that $\lim _{n \rightarrow \infty}\left\|z_{n}-u_{n}\right\|=0$, since $\lim _{k \rightarrow \infty} x_{n_{k}}=q$, thus $\lim _{k \rightarrow \infty} z_{n_{k}}=q$ and $\lim _{k \rightarrow \infty} u_{n_{k}}=q$. Hence letting $k \rightarrow \infty$, we have $\langle v-q, u\rangle \geq 0$.

Since $T$ is maximal monotone, we have $q \in T^{-1} 0$, and hence $q \in(\mathcal{V I P})_{\left(\sum_{i=1}^{N} c_{i} B_{i}, \mathcal{C}\right)}=$ $\cap_{i=1}^{N}(\mathcal{V I P})_{\left(B_{i}, \mathcal{C}\right)}$. We finally deduce that

$$
q \in \Gamma=\left(\cap_{n=1}^{\infty} F\left(S_{n}\right)\right) \cap \Omega \cap\left(\cap_{i=1}^{N}(\mathcal{V I \mathcal { P }})_{B_{i}, \mathcal{C}}\right)
$$

Next, we show that $\left\{x_{n}\right\}$ converges strongly to $q \in \Gamma$.

Indeed, as $q \in \Gamma$, we have

$$
\begin{aligned}
\left\|x_{n+1}-q\right\|^{2} & =\left\|\gamma_{n}\left(P_{\mathcal{C}_{n+1}} x_{1}-q\right)+\left(1-\gamma_{n}\right)\left(y_{n}-q\right)\right\|^{2} \\
& \leq \gamma_{n}\left\|P_{\mathcal{C}_{n+1}} x_{1}-q\right\|^{2}+\left(1-\gamma_{n}\right)\left\|y_{n}-q\right\|^{2} \\
& \leq\left\|P_{\mathcal{C}_{n+1}} x_{1}-q\right\|^{2}+\left(1-\gamma_{n}\right)\left\|x_{n}-q\right\|^{2} \\
& =\left(1-\gamma_{n}\right)\left\|x_{n}-q\right\|^{2}+\delta_{n} ;
\end{aligned}
$$

where $\delta_{n}=\left\|P_{\mathcal{C}_{n+1}} x_{1}-q\right\|^{2}$. Since

$$
\left\{\begin{array}{l}
\sum_{n=1}^{\infty} \gamma_{n}=\infty \\
\sum_{n=1}^{\infty} \delta_{n}=\sum_{n=1}^{\infty}\left\|P_{\mathcal{C}_{n+1}} x_{1}-q\right\|^{2}<\infty .
\end{array}\right.
$$

Thus all the conditions of Lemma 2.7 are satisfied. Hence we deduce that $\lim _{n \rightarrow \infty} x_{n}=q \in \Gamma$. This completes the proof. 


\section{Numerical example}

To verify the theoretical assertions, we consider the following example. All codes were written in Matlab. Let $\mathcal{H}_{1}=\mathcal{H}_{2}=\mathbb{R}$, and let $\mathcal{C}=[0,30]$ and $\left.\left.\mathcal{Q}=\right]-\infty, 0\right]$ two closed convex subsets of $\mathbb{R}$. For all $i \in\{1, \ldots, N\}$, we define $A$ and $S_{i}$ as follows:

$$
\begin{aligned}
A: \mathcal{H}_{1} & \longrightarrow \mathcal{H}_{1} \\
x & \longrightarrow 3 x
\end{aligned}
$$

and

$$
\begin{aligned}
S_{i}: \quad & \mathcal{C} \longrightarrow \mathcal{C} \\
x & \longrightarrow \frac{x}{10 i}
\end{aligned}
$$

and

$$
\begin{aligned}
B_{i}: \quad & \mathcal{C} \longrightarrow \mathcal{H}_{1} \\
& x \longrightarrow \frac{i x}{2} .
\end{aligned}
$$

It is obvious that $A$ is linear bounded and $S_{i}$ is nonexpansive. Since $S_{i}(0)=0$ then $F\left(S_{i}\right)=\{0\}$. We define the bifunctions $F_{i}$ and $G_{i}$ by:

$$
\begin{aligned}
F_{i}: \quad & \mathcal{C} \times \mathcal{C} \\
(u, v) & \longrightarrow \mathcal{H}_{1} \\
& \longrightarrow F_{i}(u, v)=i(u+1)(v-u)
\end{aligned}
$$

and

$$
\begin{aligned}
G_{i}: & \mathcal{Q} \times \mathcal{Q} \longrightarrow \mathcal{H}_{2} \\
& (x, y) \longrightarrow G_{i}(x, y)=i(x-10)(y-x)
\end{aligned}
$$

We define $a_{i}, b_{i}$ and $c_{i}$, as follows :

$$
\begin{aligned}
& a_{i}=\frac{3}{4^{i}}+\frac{1}{4^{N} N} \\
& b_{i}=c_{i}=\frac{8}{9^{i}}+\frac{1}{9^{N} N} .
\end{aligned}
$$

Let $r_{n}=\frac{n}{n+1}, s_{n}=\frac{n}{2 n+3}, \lambda_{n}=\frac{1}{n+1}, \alpha_{n}=\frac{1}{n}, \gamma_{n}=\frac{1}{n+1}$, and $\gamma=\frac{1}{11}$. It is easy to see that $F_{i}, G_{i}, r_{n}, s_{n}, \lambda_{n}, \alpha_{n}, \gamma_{n}$, and $\gamma$ are satisfying all conditions of Algorithm 3.1. In order to simplify the notation we use $\sigma=\sum_{i=1}^{N} i b_{i}=\sum_{i=1}^{N} i c_{i}$ and $\rho=\sum_{i=1}^{N} i a_{i}$. 
First, we compute $v_{n}=T_{s_{n}}^{\sum_{i=1}^{N} b_{i} G_{i}} A x_{n}$, for every $x_{n} \in \mathcal{C}, v_{n} \in \mathcal{Q}$ such that

$$
\sum_{i=1}^{N} b_{i} G_{i}\left(v_{n}, y\right)+\frac{1}{s_{n}}\left\langle y-v_{n}, v_{n}-A x_{n}\right\rangle \geq 0 .
$$

Clearly

$$
\begin{aligned}
& \Longleftrightarrow \sigma\left(v_{n}-10\right)\left(y-v_{n}\right)+\frac{1}{s_{n}}\left\langle y-v_{n}, v_{n}-A x_{n}\right\rangle \geq 0, \\
& \Longleftrightarrow s_{n} \sigma\left(v_{n}-10\right)\left(y-v_{n}\right)+\left(y-v_{n}\right)\left(v_{n}-3 x_{n}\right) \geq 0, \\
& \Longleftrightarrow\left(y-v_{n}\right)\left(\sigma s_{n}\left(v_{n}-10\right)+\left(v_{n}-3 x_{n}\right)\right) \geq 0, \\
& \Longleftrightarrow\left(y-v_{n}\right)\left(v_{n}\left(\sigma s_{n}+1\right)-\left(10 \sigma s_{n}+3 x_{n}\right)\right) \geq 0,
\end{aligned}
$$

since $T_{s_{n}}^{\sum_{i=1}^{N} b_{i} G_{i}}$ is a single-valued, then $v_{n}=\frac{10 \sigma s_{n}+3 x_{n}}{\sigma s_{n}+1}$, which holds

$$
T_{s_{n}}^{\sum_{i=1}^{N} b_{i} G_{i}} A x_{n}=\frac{10 \sigma s_{n}+3 x_{n}}{\sigma s_{n}+1} .
$$

Now, we determine $w \in \mathcal{C}$, such that $w=x_{n}-\gamma A^{*}\left(I-T_{s_{n}}^{\sum_{i=1}^{N} b_{i} G_{i}}\right) A x_{n}$, then

$$
w=x_{n}-\gamma\left(9 x_{n}-3 \frac{10 \sigma s_{n}+3 x_{n}}{\sigma s_{n}+1}\right) .
$$

In order to compute $u_{n}=T_{r_{n}}^{\sum_{i=1}^{N} a_{i} F_{i}} w$, we will find $u_{n} \in \mathcal{C}$ which satisfies

$$
\sum_{i=1}^{N} a_{i} F_{i}\left(u_{n}, z\right)+\frac{1}{r_{n}}\left\langle z-u_{n}, u_{n}-w\right\rangle \geq 0 \quad \forall z \in \mathcal{C}
$$

which is equivalent to

$$
\begin{aligned}
& \Longleftrightarrow \rho r_{n}\left(u_{n}+1\right)\left(z-u_{n}\right)+\left(z-u_{n}\right)\left(u_{n}-w\right) \geq 0, \\
& \Longleftrightarrow\left(z-u_{n}\right)\left(\rho r_{n}\left(u_{n}+1\right)+\left(u_{n}-w\right)\right) \geq 0, \\
& \Longleftrightarrow\left(z-u_{n}\right)\left(u_{n}\left(\rho r_{n}+1\right)-\left(w-\rho r_{n}\right)\right) \geq 0 .
\end{aligned}
$$

Thus, we get $u_{n}=\frac{w-\rho r_{n}}{\rho r_{n}+1}$, which holds

$$
u_{n}=\frac{1-9 \gamma}{\rho r_{n}+1} x_{n}+\frac{3 \gamma\left(10 \sigma s_{n}+3 x_{n}\right)}{\left(\sigma s_{n}+1\right)\left(\rho r_{n}+1\right)}-\frac{\rho r_{n}}{\rho r_{n}+1} .
$$

Then

$$
z_{n}=P_{\mathcal{C}}\left[u_{n}-\lambda_{n} \sigma \frac{u_{n}}{2}\right]
$$




$$
y_{n}=\frac{\alpha_{n}}{10 n} x_{n}+\left(1-\alpha_{n}\right) z_{n}
$$

and thus

$$
x_{n+1}=\gamma_{n} P_{\mathcal{C}_{n+1}} x_{1}+\left(1-\gamma_{n}\right) y_{n},
$$

since for $x_{1} \in \mathcal{C}=\mathcal{C}_{1}$, we get $0 \leq y_{1} \leq x_{1} \leq 30$, then

$$
\mathcal{C}_{2}=\left\{p \in \mathcal{C}:\left|y_{1}-p\right| \leq\left|x_{1}-p\right|\right\}=\left[0, \frac{y_{1}+x_{1}}{2}\right]
$$

it can be clearly seen that $\frac{y_{1}+x_{1}}{2} \leq x_{1}$, which implies $x_{2}=P_{\mathcal{C}_{2}} x_{1}=\frac{y_{n, 1}+x_{1}}{2}$. Therefore by the same process we easily get $\mathcal{C}_{n+1}=\left[0, \frac{y_{n}+x_{n}}{2}\right]$, consequently $P_{\mathcal{C}_{n+1}} x_{1}=\frac{y_{n}+x_{n}}{2}$. Thus $x_{n+1}$ can be rewrited as follows

$$
x_{n+1}=\gamma_{n} \frac{y_{n}+x_{n}}{2}+\left(1-\gamma_{n}\right) y_{n} .
$$

Hence, the new form of the algorithm is given by:

$$
\left\{\begin{array}{l}
u_{n}=\frac{1-9 \gamma}{\rho r_{n}+1} x_{n}+\frac{3 \gamma\left(10 \sigma s_{n}+3 x_{n}\right)}{\left(\sigma s_{n}+1\right)\left(\rho r_{n}+1\right)}-\frac{\rho r_{n}}{\rho r_{n}+1}, \\
z_{n}=P_{\mathcal{C}}\left[u_{n}-\lambda_{n} \sigma \frac{u_{n}}{2}\right] \\
y_{n}=\frac{\alpha_{n}}{10} x_{n}+\left(1-\alpha_{n}\right) z_{n}, \\
x_{n+1}=\gamma_{n} \frac{y_{n}+x_{n}}{2}+\left(1-\gamma_{n}\right) y_{n}, \quad n \geq 1 .
\end{array}\right.
$$

\begin{tabular}{ccccc|cccc}
\multicolumn{5}{c}{ Algorithm 3.1 } & \multicolumn{4}{c|}{ Algorithm 3.2 } \\
\cline { 2 - 8 } $\mathbf{n}$ & $u_{n}$ & $z_{n}$ & $y_{n}$ & $x_{n}$ & $u_{n}$ & $z_{n}$ & $y_{n}$ & $x_{n}$ \\
\hline $\mathbf{1}$ & 4.998900 & 3.593000 & 1.000000 & 10.000000 & 5.727300 & 4.295500 & 1.000000 & 10.000000 \\
$\mathbf{2}$ & 1.258800 & 1.022800 & 0.592630 & 3.250000 & 1.559100 & 1.299200 & 0.730870 & 3.250000 \\
$\mathbf{3}$ & 0.274130 & 0.235580 & 0.168560 & 1.035500 & 0.484100 & 0.423580 & 0.295180 & 1.150700 \\
$\mathbf{4}$ & -0.030834 & 0.000000 & 0.001731 & 0.276930 & 0.134250 & 0.120830 & 0.093135 & 0.402120 \\
$\mathbf{5}$ & -0.125720 & 0.000000 & 0.000117 & 0.029251 & 0.010956 & 0.0100430 & 0.008531 & 0.124030 \\
$\mathbf{6}$ & -0.137460 & 0.000000 & 0.000007 & 0.002545 & -0.034467 & 0.000000 & 0.000050 & 0.018156 \\
$\mathbf{7}$ & -0.139900 & 0.000000 & 0.000000 & 0.000188 & -0.041879 & 0.000000 & 0.000003 & 0.001344 \\
$\mathbf{8}$ & -0.141200 & 0.000000 & 0.000000 & 0.000012 & -0.042746 & 0.000000 & 0.000000 & 0.000086 \\
$\mathbf{9}$ & -0.142190 & 0.000000 & 0.000000 & 0.000000 & -0.043060 & 0.000000 & 0.000000 & 0.000005 \\
$\mathbf{1 0}$ & -0.142980 & 0.000000 & 0.000000 & 0.000000 & -0.043290 & 0.000000 & 0.000000 & 0.000000 \\
\hline
\end{tabular}

Table 1: the values of $\left\{u_{n}\right\},\left\{z_{n}\right\},\left\{y_{n}\right\}$ and $\left\{x_{n}\right\}$ with initial value $x_{1}=10$.

Figures 1 and 2 clearly show the behavior of the sequence $\left\{x_{n}\right\}$ generated by the Algorithm 3.1, which converges to the same solution i.e., $0 \in \Gamma=\left(\cap_{i=1}^{\infty} F\left(S_{i}\right)\right) \cap \Omega \cap\left(\cap_{i=1}^{N}(\mathcal{V I \mathcal { P }})_{B_{i}, \mathcal{C}}\right)$. 

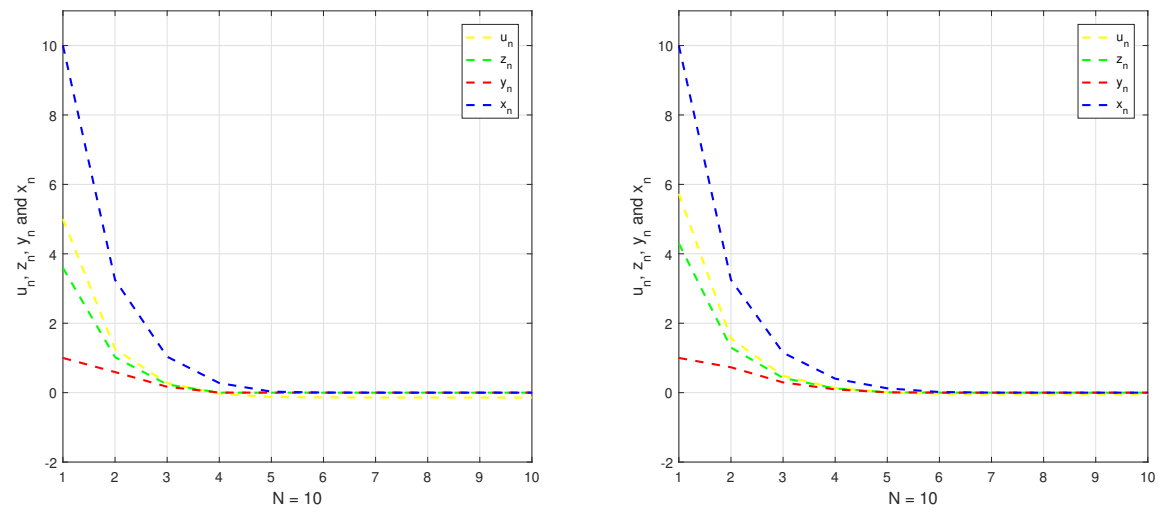

Figure 1: Convergence of $\left\{u_{n}\right\},\left\{z_{n}\right\},\left\{y_{n}\right\}$ and $\left\{x_{n}\right\}$ with initial value $x_{1}=10$, for Algorithm 3.1 and Algorithm 3.2.

And the algorithm 3.2, which converges to 0. i.e., $0 \in \Gamma=\left(\cap_{i=1}^{\infty} F\left(S_{i}\right)\right) \cap \Omega \cap(\mathcal{V I P})_{B, \mathcal{C}}$.

Figures 3 and 4 show again that the sequence $x_{n}$ generated by the Algorithm 3.1. converges to 0 , where $0 \in \Gamma=\left(\cap_{i=1}^{\infty} F\left(S_{i}\right)\right) \cap \Omega \cap\left(\cap_{i=1}^{N}(\mathcal{V I P})_{B_{i}, \mathcal{C}}\right)$. Similary Algorithm 3.2 converges to 0 , where $0 \in \Gamma=\left(\cap_{i=1}^{\infty} F\left(S_{i}\right)\right) \cap \Omega \cap(\mathcal{V I P})_{B, \mathcal{C}}$.

In the following we compare the proposed method with those in [20] and [13].

Remark 1. Table 3 and Figure 5, show that the sequence $\left\{x_{n}\right\}$ converges faster than those in [20] and [13].

\section{Conclusions}

In this paper, we suggested and analyzed an iterative method for finding the approximate element of the common set of solutions of the combination of the split equilibrium problem (2.1)-(2.2), a finite family of nonexpansive mapping (2.3) and the combination of variational inequality problem (2.4) in real Hilbert spaces. We proved that the sequences generated by the proposed iterative method converge strongly to a common solution to these problems. We also discussed a numerical example to demonstrate the applicability of the iterative algorithm. The method and results presented in this paper can be viewed as a refinement and improvement of some existing methods for solving a variational inequality problem and a split equilibrium problem. 

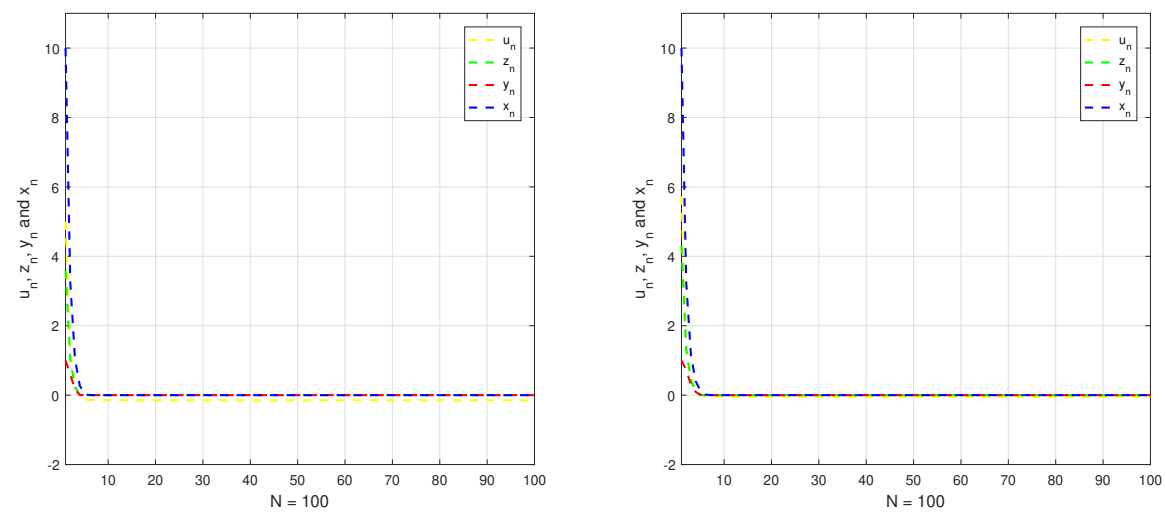

Figure 2: Convergence of $\left\{u_{n}\right\},\left\{z_{n}\right\},\left\{y_{n}\right\}$ and $\left\{x_{n}\right\}$ with initial value $x_{1}=10$, for Algorithm 3.1 and Algorithm 3.2.
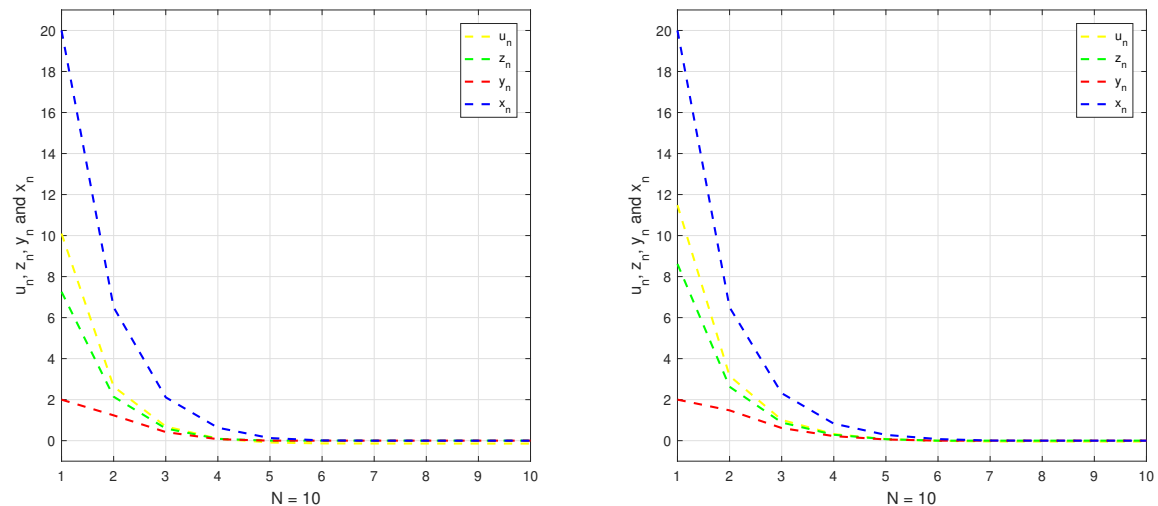

Figure 3: Convergence of $\left\{u_{n}\right\},\left\{z_{n}\right\},\left\{y_{n}\right\}$ and $\left\{x_{n}\right\}$ with initial value $x_{1}=20$, for Algorithm 3.1 and Algorithm 3.2. 


\begin{tabular}{ccccc|cccc}
\multicolumn{5}{c}{ Algorithm 3.1 } & \multicolumn{4}{c}{ Algorithm 3.2 } \\
\cline { 2 - 9 } $\mathbf{n}$ & $u_{n}$ & $z_{n}$ & $y_{n}$ & $x_{n}$ & $u_{n}$ & $z_{n}$ & $y_{n}$ & $x_{n}$ \\
\hline $\mathbf{1}$ & 10.097000 & 7.257400 & 2.000000 & 20.000000 & 11.485000 & 8.613600 & 2.000000 & 20.000000 \\
$\mathbf{2}$ & 2.636900 & 2.142500 & 1.233800 & 6.500000 & 3.154500 & 2.628800 & 1.476900 & 6.500000 \\
$\mathbf{3}$ & 0.692060 & 0.594740 & 0.419950 & 2.111500 & 1.012900 & 0.886280 & 0.616570 & 2.314100 \\
$\mathbf{4}$ & 0.099939 & 0.088696 & 0.070468 & 0.631390 & 0.319560 & 0.287600 & 0.220880 & 0.828760 \\
$\mathbf{5}$ & -0.091017 & 0.000000 & 0.000506 & 0.126560 & 0.077398 & 0.070948 & 0.057885 & 0.281670 \\
$\mathbf{6}$ & -0.134510 & 0.000000 & 0.000003 & 0.011011 & -0.010381 & 0.000000 & 0.000213 & 0.076534 \\
$\mathbf{7}$ & -0.139680 & 0.000000 & 0.000002 & 0.000815 & -0.040124 & 0.000000 & 0.000011 & 0.005664 \\
$\mathbf{8}$ & -0.141190 & 0.000000 & 0.000000 & 0.000052 & -0.042634 & 0.000000 & 0.000000 & 0.000365 \\
$\mathbf{9}$ & -0.142190 & 0.000000 & 0.000000 & 0.000003 & -0.043054 & 0.000000 & 0.000000 & 0.000020 \\
$\mathbf{1 0}$ & -0.142980 & 0.000000 & 0.000000 & 0.000000 & -0.043290 & 0.000000 & 0.000000 & 0.000001 \\
\hline
\end{tabular}

Table 2: The values of $\left\{u_{n}\right\},\left\{z_{n}\right\},\left\{y_{n}\right\}$ and $\left\{x_{n}\right\}$ with initial value $x_{1}=20$.

\begin{tabular}{cc|c|c}
\hline & Algorithm 3.1 & Algorithm [20] & Algorithm [13] \\
\cline { 2 - 4 } $\mathbf{n}$ & $x_{n}$ & $x_{n}$ & $x_{n}$ \\
\hline $\mathbf{1}$ & 5.000000 & 5.000000 & 5.000000 \\
$\mathbf{2}$ & 1.625000 & 2.500000 & 5.000000 \\
$\mathbf{3}$ & 0.497560 & 1.250000 & 3.792000 \\
$\mathbf{4}$ & 0.099701 & 0.625000 & 2.571000 \\
$\mathbf{5}$ & 0.010531 & 0.312500 & 1.639600 \\
$\mathbf{6}$ & 0.000916 & 0.156250 & 1.005600 \\
$\mathbf{7}$ & 0.000068 & 0.078125 & 0.599980 \\
$\mathbf{8}$ & 0.000004 & 0.039063 & 0.350430 \\
$\mathbf{9}$ & 0.000000 & 0.019531 & 0.200930 \\
$\mathbf{1 0}$ & 0.000000 & 0.009766 & 0.113100 \\
\hline
\end{tabular}

Table 3: the values $\left\{x_{n}\right\}$ with initial value $x_{1}=5$. with three different methods.

\section{References}

[1] E. Blum and W. Oettli, From optimization and variational inequalities to equilibrium problems, The Mathematics Student, 63 (1994), 123-145.

[2] A. Bnouhachem, An iterative algorithm for system of generalized equilibrium problems and fixed point problem, Fix. Point Th. Appl. 2014(235) (2014), 1-21.

[3] A. Bnouhachem, A modified projection method for a common solution of a system of variational inequalities, a split equilibrium problem and a hierarchical fixed-point problem, Fix. Point Th. Appl. 2014(22) (2014), 1-25.

[4] A. Bnouhachem, S. Al-Homidan and Q. H. Ansari, An iterative method for common solutions 

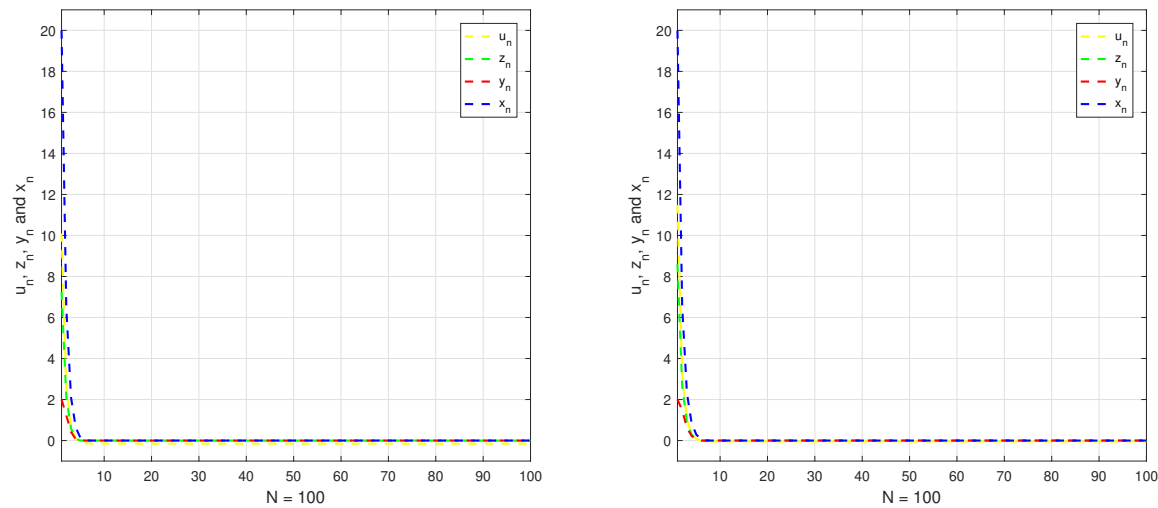

Figure 4: Convergence of $\left\{u_{n}\right\},\left\{z_{n}\right\},\left\{y_{n}\right\}$ and $\left\{x_{n}\right\}$ with initial value $x_{1}=20$, for Algorithm 3.1 and Algorithm 3.2.
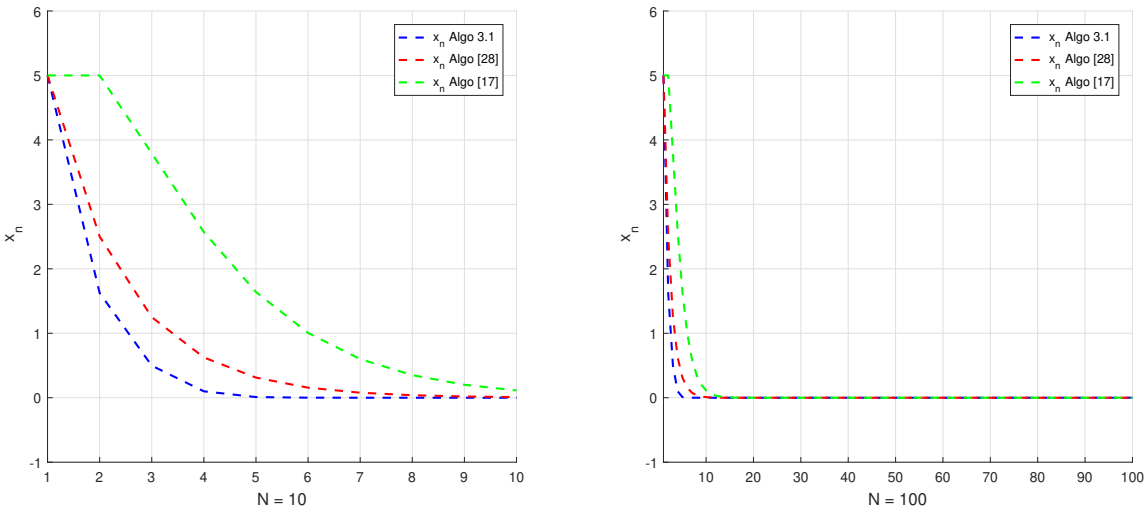

Figure 5: Convergence of $\left\{x_{n}\right\}$ with initial value $x_{1}=5$, with different number of iterations.

of equilibrium problems and hierarchical fixed point problems, Fix. Point Th. Appl. 194 (2014), 1-21.

[5] A. Bnouhachem, A hybrid iterative method for a combination of equilibria problem, a combination of variational inequality problems and a hierarchical fixed point problem, Fix. Point Th. Appl. 163(2014), 1-29.

[6] A. Bnouhachem, Strong convergence algorithm for approximating the common solutions of a variational inequality, a mixed equilibrium problem and a hierarchical fixed-point problem, J. Inequal. Appl. 154 (2014), 1-24.

[7] L. C. Ceng and J. C. Yao, A hybrid iterative scheme for mixed equilibrium problems and fixed point problems, J. Comput. Appl. Math. 214(1) (2008), 186-201. 
[8] S. S. Chang, H. W. Joseph Lee and C. K. Chan, A new method for solving equilibrium problem fixed point problem and variational inequality problem with application to optimization, Nonlinear Anal. 70 (2009), 3307-3319.

[9] C. E. Chidume and M. O. Nnakwe, Iterative algorithms for split variational inequalities and generalized split feasibility problems with applications, J. Nonlinear Var. Anal. 3 (2019), 127140.

[10] F. Cianciaruso, G. Marino, L. Muglia and Y. Yao, A hybrid projection algorithm for finding solutions of mixed equilibrium problem and variational inequality problem, Fix. Point Th. Appl. 2010 (2010), Article ID 383740, 19 pages.

[11] P. L. Combettes and S. A. Hirstoaga, Equilibrium programming using proximal like algorithms, Math. Prog. 78 (1997), 29-41.

[12] P. L. Combettes and S. A. Hirstoaga, Equilibrium programming in Hilbert spaces, J. Nonlinear Convex Anal. 6 (2005), 117-136.

[13] J. Deepho, W. Kumam and P. Kumam, A new hybrid projection algorithm for solving the split generalized equilibrium problems and the system of variational inequality problems, J. Math. Model. Algor. 13(4) (2014), 405-423.

[14] J. Deepho, J. M. Moreno and P. Kumam, A viscosity of Cesaro mean approximation method for split generalized equilibrium, variational inequality and fixed point problems, J. Nonlinear Sci. Appl. 9, 1475-1496 (2016).

[15] M. Farid, The subgradient extragradient method for solving mixed equilibrium problems and fixed point problems in Hilbert spaces, J. Appl. Numer. Optim. 1 (2019), 335-345.

[16] K. R. Kazmi and S. H. Rizvi, Iterative approximation of a common solution of a split generalized equilibrium problem and a fixed point problem for nonexpansive semigroup, Math. Sci. 7(1) (2013), 1-10.

[17] K. R. Kazmi and S. H. Rizvi, Iterative approximation of a common solution of a split equilibrium problem, a variational inequality problem and a fixed point problem, J. Egypt. Math. Society 21(1) (2013), 44-51.

[18] A. Latif, L. C. Ceng and Q. H. Ansari, Multi-step hybrid viscosity method for systems of variational inequalities defined over sets of solutions of equilibrium problem and fixed point problems, Fix. Point Th. Appl. 2012(186) (2012), 1-26. 
[19] C. Marino, L. Muglia and J. C. Yao, Viscosity methods for common solutions of equilibrium and variational inequality problems via multi-step iterative algorithms and common fixed points. Nonlinear Anal. 75(4) (2012), 1787-1798.

[20] W. Phuengrattana and K. Lerkchaiyaphum, On solving the split generalized equilibrium problem and the fixed point problem for a countable family of nonexpansive multivalued mappings, Fix. Point Th. Appl. 2018(1) (2018), 1-17.

[21] X. Qin, M. Shang and Y. Su, A general iterative method for equilibrium problem and fixed point problem in Hilbert spaces, Nonlinear Anal. 69(11) (2008), 3897-3909.

[22] S. Reich and S. Sabach, Three strong convergence theorems regarding iterative methods for solving equilibrium problems in reflexive Banach spaces, optimization theory and related topics, Contemp. Math. 568 (2012), 225-240.

[23] R. T. Rockafellar, On the maximality of sums nonlinear monotone operators, Trans. Amer. Math. Soc. 149 (1970), 75-88 (1970).

[24] G. Stampacchia, Formes bilineaires coercitives sur les ensembles convexs, C. R. Acad. Sci. Paris. 258 (1964), 4413-4416.

[25] S. Suwannaut and A. Kangtunyakarn, The combination of the set of solutions of equilibrium problem for convergence theorem of the set of fixed points of strictly pseudo-contractive mappings and variational inequalities problem, Fix. Point Th. Appl. 291 (2013), 1-26.

[26] S. Takahashi and W. Takahashi, Strong convergence theorem for a generalized equilibrium problem and a nonexpansive mapping in a Hilbert space, Nonlinear Anal. 69(3) (2008), 10251033.

[27] H. K. Xu, Iterative algorithms for nonlinear operators, J. London Math. Soc. 66(1) (2002), 240-256.

[28] J. Zhao, D. Hou and H. Zong, Several iterative algorithms for solving the multiple-set split common fixed-point problem of averaged operators, J. Nonlinear Funct. Anal. 2019 (2019), Article ID 39.

Ihssane Hay Equipe MAISI, Ibn Zohr University, ENSA, BP 1136, Agadir, Morocco.

E-mail: ihssane.hay@edu.uiz.ac.ma

Abdellah Bnouhachem Equipe MAISI, Ibn Zohr University, ENSA, BP 1136, Agadir, Morocco. 
E-mail: babedallah@yahoo.com

Themistocles M.Rassias Department of Mathematics, National Technical University of Athens Zografou Campus 157 80, Athens, Greece.

E-mail: trassias@math.ntua.gr 